\title{
A novel treatment of the proton-proton Coulomb force in elastic proton-deuteron Faddeev calculations
}

\author{
H. Witała, R. Skibiński, and J. Golak \\ M. Smoluchowski Institute of Physics, \\ Jagiellonian University, PL-30059 Kraków, Poland \\ W. Glöckle \\ Institut für theoretische Physik II, Ruhr-Universität Bochum, D-44780 Bochum, Germany
}

(Dated: June 2, 2021)

\begin{abstract}
We propose a novel approach to incorporate the proton-proton (pp) Coulomb force into the three-nucleon (3N) Faddeev calculations. The main new ingredient is a 3-dimensional screened pp Coulomb t-matrix obtained by a numerical solution of the 3-dimensional Lippmann-Schwinger (LS) equation. We demonstrate numerically and provide analytical insight that the elastic proton-deuteron (pd) observables can be determined directly from the resulting on shell $3 \mathrm{~N}$ amplitude increasing the screening radius. The screening limit exists without the need of renormalisation not only for observables but for the elastic pd amplitude itself.

PACS numbers: 21.45.-v, 21.45.Bc, 25.10.+s, 25.40.Cm
\end{abstract}




\section{INTRODUCTION}

The inclusion of the Coulomb force into the analysis of nuclear reactions with more than 2 nucleons is a long standing problem. The main reason is the long-range nature of the Coulomb force which prevents the application of the standard techniques developed for short-range interactions. One possible way to avoid the difficulties including the Coulomb force is to use a screened Coulomb interaction and to reach the pure Coulomb limit through application of a renormalisation procedure [1, 2, 3, 4].

The problem is especially timely to be solved when considering the interaction of protons with deuterons below the pion production threshold. For this 3N system using the Faddeev scheme highprecision numerical predictions for different observables in elastic proton-deuteron (pd) scattering and for the deuteron breakup reaction are being obtained [5], however, only under the restriction to short-ranged nuclear interactions. The high quality of the available pd data for both processes requires, however, in the theoretical analysis the inclusion of the Coulomb force into the calculations. Furthermore the seminal progress [6] in the development of nuclear forces in chiral effective field theory calls also for a very precise solution of the pd scattering equations to test unambiguously these new dynamical ingredients. This test can only be completely satisfactory if the pp Coulomb force is perfectly under control.

For the elastic pd scattering first calculations, with modern nuclear forces and the Coulomb force included, have been achieved in a variational hyperspherical harmonic approach [7]. Only recently the inclusion of the Coulomb force became possible also for the pd breakup reaction [8]. In [8], contrary to [7] where the exact Coulomb force in coordinate representation has been used directly, a screened pp Coulomb force has been applied in momentum space and in a partial wave basis. In order to get the final predictions which can be compared to the data, the limit to the unscreened situation has been performed numerically applying a renormalization to the resulting on-shell amplitudes [8, 9]. This allowed for the first time to analyze high-precision pd breakup data and provided a significant improvement of data description in cases where the Coulomb force plays an important role [10].

However, in spite of that substantial progress some important questions remained unanswered. One concerns directly the results of these calculations for two kinematically complete breakup geometries: the pp quasi-free-scattering (QFS) configuration, in which the not detected neutron is at rest in the laboratory system, and the space-star (SST) geometry, in which all 3 outgoing nucleons have the same momenta (magnitudes) in the plane which in the $3 \mathrm{~N}$ c.m. system is 
perpendicular to the incoming nucleon momentum. The theoretical predictions based on nuclear forces only show, that the cross sections for QFS and SST are quite stable against changes of the underlying interactions, including also three-nucleon forces [5]. At energies below $\approx 20 \mathrm{MeV}$ theory underestimates the SST pd cross sections by $\approx 10 \%$, and overestimates the pp QFS cross sections by $\approx 20 \%$, respectively [5, 8]. With increasing energy the theoretical cross sections come close to the data, which indicates that the pp Coulomb force is very probably responsible for these low energy discrepancies. However, the Coulomb force effects found in [8] are practically negligible for the pd SST configuration and only slightly improve the description of the pp QFS data [11, 12, 13, 14, 15].

This inability to understand the pp QFS and pd SST cross sections calls for reconsidering the inclusion of the Coulomb force into momentum space Faddeev calculations. One main concern in such type of calculations is the application of a partial wave decomposition to the long-ranged Coulomb force. Even when screening is applied it seems reasonable to treat from the beginning the screened pp Coulomb t-matrix without partial wave decomposition because the required limit of vanishing screening leads necessarily to a drastic increase of the number of partial wave states involved. As an example we provide numbers for the exponential screening $e^{-\left(\frac{r}{R}\right)^{n}}$. Taking the screening radius $R=20 \mathrm{fm}$ and $n=4$ requires all $l \leq l_{\max }=10$ partial wave states to reproduce the 3-dimensional pp screened Coulomb t-matrix at $E_{p}^{l a b}=13 \mathrm{MeV}$. Increasing the screening radius to $R=120 \mathrm{fm}$ requires $l_{\max } \approx 50$ which is a big numerical challenge. Even more that would lead to an explosion of the number of $3 \mathrm{~N}$ partial waves required for convergence.

Another problem concerns the treatment of the pp Coulomb interaction in its proper coordinate, which is the relative proton-proton distance, throughout the calculations. Any deviation from this restriction can cause effects which are difficult to estimate.

In the present paper we propose a novel approach taking both concerns into account to incorporate the pp Coulomb force into the momentum space Faddeev calculations, in which we apply directly the 3-dimensional screened pp Coulomb t-matrix without relying on a partial wave decomposition. 3-dimensional solutions of the LS equation for different screening radii are used to approach the final predictions. We demonstrate, that even the physical elastic pd scattering amplitude has a well defined screening limit and does not require renormalisation. In contrast in case of pp scattering the scattering amplitude requires renormalisation in the screening limit which, however, has not to be applied when only observables have to be addressed [16].

In section II we present details of the formalism and in section III the physical amplitude for elastic pd scattering. The screening limit is discussed in section IV and the results shown in section 
V] The summary is given in section VI, In various appendices detailed expressions for different kernel elements appearing in the Faddeev equation with the screened Coulomb force are included and different terms contributing to the on-shell elastic scattering amplitude are given.

\section{FADDEEV EQUATIONS WITH SCREENED PP COULOMB FORCE}

The $3 \mathrm{~N}$ pd system can be regarded in a particle basis or in the isospin basis. The corresponding completeness relations are

$$
\begin{gathered}
|p n p><p n p|+|p p n><p p n|+|n p p><n p p|=1, \\
\sum_{t T}|(t 1 / 2) T-1 / 2><(t 1 / 2) T-1 / 2|=1 .
\end{gathered}
$$

In (2) $\mathrm{t}$ is the 2-body isospin which together with the isospin $\frac{1}{2}$ of the third particle is coupled to the total isospin $\mathrm{T}$. We use the convention that the proton (neutron) has the isospin magnetic quantum number $-\frac{1}{2}\left(\frac{1}{2}\right)$. Since NN forces are usually given in an isospin basis we stick to that formulation also in the $3 \mathrm{~N}$ system. Then a general $\mathrm{NN}$ force acts in the $3 \mathrm{~N}$ system as

$$
V=\sum_{t, T} \sum_{t^{\prime}, T^{\prime}}\left|(t 1 / 2) T M_{T}><(t 1 / 2) T M_{T}\right| V\left|\left(t^{\prime} 1 / 2\right) T^{\prime} M_{T}><\left(t^{\prime} 1 / 2\right) T^{\prime} M_{T}\right| .
$$

Here we assume that $\mathrm{t}$ is conserved, which in the pd system leads to [17]

$$
\begin{aligned}
V & =\left|(01 / 2) 1 / 2-1 / 2>V_{n p}^{00}<(01 / 2) 1 / 2-1 / 2\right| \\
& +\left|(11 / 2) 1 / 2-1 / 2>\left(1 / 3 V_{n p}^{10}+2 / 3 V_{p p}^{1-1}\right)<(11 / 2) 1 / 2-1 / 2\right| \\
& +\left|(11 / 2) 1 / 2-1 / 2>\frac{\sqrt{2}}{3}\left(V_{n p}^{10}-V_{p p}^{1-1}\right)<(11 / 2) 3 / 2-1 / 2\right| \\
& +\left|(11 / 2) 3 / 2-1 / 2>\left(2 / 3 V_{n p}^{10}+1 / 3 V_{p p}^{1-1}\right)<(11 / 2) 3 / 2-1 / 2\right| \\
& +\left|(11 / 2) 3 / 2-1 / 2>\frac{\sqrt{2}}{3}\left(V_{n p}^{10}-V_{p p}^{1-1}\right)<(11 / 2) 1 / 2-1 / 2\right| .
\end{aligned}
$$

We defined in the usual manner

$$
\begin{aligned}
V_{n p}^{t 0} & =<t 0|V| t 0> \\
V_{p p}^{1-1} & =<1-1|V| 1-1>.
\end{aligned}
$$

The latter decomposes into the strong part and the pure Coulomb part, which we assume to be screened and parametrised by some parameter $R$

$$
V_{p p}^{1-1}=V_{p p}^{\text {strong }}+V_{p p}^{c R} .
$$


Eq.(4) exhibits that charge independence breaking leads necessarily to a coupling of $T=1 / 2$ and $T=3 / 2$ states and the complete action of the Coulomb force also requires the presence of both total isospin states. Exactly the same decomposition holds for the $2 \mathrm{~N}$ t-operator $\hat{t}$

$$
\begin{aligned}
\hat{t} & =\left|(01 / 2) 1 / 2-1 / 2>t_{n p}^{00}<(01 / 2) 1 / 2-1 / 2\right| \\
& +\left|(11 / 2) 1 / 2-1 / 2>\left(1 / 3 t_{n p}^{10}+2 / 3 t_{p p}^{1-1}\right)<(11 / 2) 1 / 2-1 / 2\right| \\
& +\left|(11 / 2) 1 / 2-1 / 2>\frac{\sqrt{2}}{3}\left(t_{n p}^{10}-t_{p p}^{1-1}\right)<(11 / 2) 3 / 2-1 / 2\right| \\
& +\left|(11 / 2) 3 / 2-1 / 2>\left(2 / 3 t_{n p}^{10}+1 / 3 t_{p p}^{1-1}\right)<(11 / 2) 3 / 2-1 / 2\right| \\
& +\left|(11 / 2) 3 / 2-1 / 2>\frac{\sqrt{2}}{3}\left(t_{n p}^{10}-t_{p p}^{1-1}\right)<(11 / 2) 1 / 2-1 / 2\right| .
\end{aligned}
$$

It is not difficult to see [17] that this is consistent with the 2-body Lippmann Schwinger equation

$$
\hat{t}=V+V G_{0} \hat{t}
$$

when expanded into the $3 \mathrm{~N}$ isospin basis.

It is now convenient to define the three $3 \mathrm{~N}$ isospin states

$$
\begin{aligned}
& \left|\gamma_{0}>=\right|(01 / 2) 1 / 2-1 / 2> \\
& \left|\gamma_{1}>=\right|(11 / 2) 1 / 2-1 / 2> \\
& \left|\gamma_{2}>=\right|(11 / 2) 3 / 2-1 / 2>.
\end{aligned}
$$

Then $\hat{t}$ appears as

$$
\hat{t}=\sum_{\gamma, \gamma^{\prime}}\left|\gamma>t_{\gamma, \gamma^{\prime}}<\gamma^{\prime}\right|
$$

with

$$
t_{\gamma, \gamma^{\prime}}=\delta_{t t^{\prime}} t_{t}^{T T^{\prime}}
$$

and $t_{t}^{T T^{\prime}}$ can be read of from (8) .

We use the Faddeev equation in the form [5]

$$
T|\Phi>=\hat{t} P| \Phi>+\hat{t} P G_{0} T \mid \Phi>
$$

where $P$ is defined in terms of transposition operators, $P=P_{12} P_{23}+P_{13} P_{23}, G_{0}$ is the free $3 \mathrm{~N}$ propagator, $\mid \Phi>$ the initial state composed of a deuteron state and a momentum eigenstate of the proton. Knowing $T \mid \Phi>$ the breakup as well as the elastic pd scattering amplitudes can be gained by quadratures in the standard manner [5]. 
Expanding $T \mid \Phi>$

$$
T\left|\Phi>=\sum_{\gamma}\right| \gamma>T_{\gamma}
$$

(13) turns into

$$
T_{\gamma}=\sum_{\gamma^{\prime}} t_{\gamma \gamma^{\prime}}<\gamma^{\prime}|P| \Phi>+\sum_{\gamma^{\prime}} t_{\gamma \gamma^{\prime}}<\gamma^{\prime}\left|P G_{0}\right| \gamma^{\prime \prime}>T_{\gamma^{\prime \prime}}
$$

In a more detailed notation we use (12) and define

$$
\begin{array}{r}
T_{\gamma} \equiv T_{t}^{T} \\
<\gamma|P| \gamma^{\prime}>=\delta_{T T^{\prime}} F\left(t t^{\prime} T\right) P_{t t^{\prime}} \equiv \delta_{T T^{\prime}} P_{t t^{\prime}}^{T}
\end{array}
$$

where $P_{t t^{\prime}}$ acts only in spin-momentum space,

$$
P_{t t^{\prime}}=P_{12} P_{23}+\left.(-)^{t+t^{\prime}} P_{13} P_{23}\right|_{\text {spin-momentum }}
$$

and $F\left(t t^{\prime} T\right)$ is given by

$$
F\left(t t^{\prime} T\right)=(-)^{t^{\prime}} \sqrt{2 t+1} \sqrt{2 t^{\prime}+1} \times\left\{\begin{array}{ccc}
t & 1 / 2 & 1 / 2 \\
t^{\prime} & T & 1 / 2
\end{array}\right\} .
$$

For the convenience of the reader the nonzero values are

$$
\begin{aligned}
& F(111 / 2)=-\frac{1}{2} \\
& F(113 / 2)=1 \\
& F(101 / 2)=\frac{\sqrt{3}}{2} \\
& F(001 / 2)=-\frac{1}{2} \\
& F(011 / 2)=-\frac{\sqrt{3}}{2}
\end{aligned}
$$

This leads to the more explicit form of the coupled set of equations (15)

$$
T_{t}^{T}=t_{t}^{T \frac{1}{2}} P_{t 0}^{\frac{1}{2}} \mid \phi>+\sum_{T^{\prime}} t_{t}^{T T^{\prime}} \sum_{t^{\prime \prime}} P_{t t^{\prime \prime}}^{T^{\prime}} G_{0} T_{t^{\prime \prime}}^{T^{\prime}} .
$$

where we used the isospin independence of the free propagator $G_{0}$ and

$$
|\Phi>=| \gamma_{0}>\mid \phi>
$$


This set (25) would be the starting point for a calculation using momentum vectors instead of an angular momentum decomposition. In view of forthcoming calculations we provide that set as follows

$$
\begin{aligned}
T_{0}^{1 / 2} & =-\frac{1}{2} t_{n p}^{00} P_{00} \mid \phi>-\frac{1}{2} t_{n p}^{00} P_{00} G_{0} T_{0}^{1 / 2}-\frac{\sqrt{3}}{2} t_{n p}^{00} P_{01} G_{0} T_{1}^{1 / 2} \\
T_{1}^{1 / 2} & =\frac{\sqrt{3}}{2}\left(\frac{1}{3} t_{n p}^{10}+\frac{2}{3} t_{p p}^{1-1}\right) P_{10} \mid \phi>+\frac{\sqrt{3}}{2}\left(\frac{1}{3} t_{n p}^{10}+\frac{2}{3} t_{p p}^{1-1}\right) P_{10} G_{0} T_{0}^{1 / 2} \\
& -\frac{1}{2}\left(\frac{1}{3} t_{n p}^{10}+\frac{2}{3} t_{p p}^{1-1}\right) P_{11} G_{0} T_{1}^{1 / 2}+\frac{\sqrt{2}}{3}\left(t_{n p}^{10}-t_{p p}^{1-1}\right) P_{11} G_{0} T_{1}^{3 / 2} \\
T_{1}^{3 / 2} & =\frac{1}{\sqrt{6}}\left(t_{n p}^{10}-t_{p p}^{1-1}\right) P_{10} \mid \phi>+\frac{1}{\sqrt{6}}\left(t_{n p}^{10}-t_{p p}^{1-1}\right) P_{10} G_{0} T_{0}^{1 / 2} \\
& -\frac{1}{3 \sqrt{2}}\left(t_{n p}^{10}-t_{p p}^{1-1}\right) P_{11} G_{0} T_{1}^{1 / 2}+\left(\frac{2}{3} t_{n p}^{10}+\frac{1}{3} t_{p p}^{1-1}\right) P_{11} G_{0} T_{1}^{3 / 2}
\end{aligned}
$$

Note $t_{p p}^{1-1}$ is driven by the strong and the screened pp Coulomb force (7).

Here, however, we apply the angular momentum decomposition and define the basis states

$$
|p q a>\equiv| p q(l s) j\left(\lambda \frac{1}{2}\right) I(j I) J M>
$$

where $p, q$ are the standard Jacobi momenta, $(l s) j$ refers to the 2 -body subsystem, $\left(\lambda \frac{1}{2}\right) I$ to the third particle and $J M$ is the total angular momentum and its magnetic quantum number. In that partial wave basis the set (25) reads

$$
\begin{aligned}
<p q a \mid T_{t}^{T}> & =\sum_{a^{\prime}} \int d p^{\prime} p^{\prime 2} d q^{\prime} q^{2}<p q a\left|t_{t}^{T \frac{1}{2}}\right| p^{\prime} q^{\prime} a^{\prime}><p^{\prime} q^{\prime} a^{\prime}\left|P_{t 0}^{\frac{1}{2}}\right| \phi> \\
& +\sum_{T^{\prime} t^{\prime \prime}} \sum_{a^{\prime}} \int d p^{\prime} p^{\prime 2} d q^{\prime} q^{\prime 2}<p q a\left|t_{t}^{T T^{\prime}}\right| p^{\prime} q^{\prime} a^{\prime}><p^{\prime} q^{\prime} a^{\prime} \mid P_{t t^{\prime \prime}}^{T^{\prime}} G_{0} T_{t^{\prime \prime}}^{T \prime}>
\end{aligned}
$$

This form allows us to separate the lower partial waves in the 2-body subsystem, where the strong force acts together with the Coulomb force, from the higher partial waves, where only the pure pp Coulomb t-matrix is present.

Thus for the higher partial waves, denoted as $\mid p q a^{>}>$, we obtain

$$
\begin{aligned}
<p q a^{>} \mid T_{0}^{\frac{1}{2}}> & =0 \\
<p q a^{>} \mid T_{1}^{T}> & =\sum_{a^{\prime}} \int d p^{\prime} p^{\prime 2} d q^{\prime} q^{\prime 2}<p q a^{>}\left|t_{1 c}^{T \frac{1}{2}}\right|\left|p^{\prime} q^{\prime} a^{\prime}><p^{\prime} q^{\prime} a^{\prime}\right| P_{10}^{\frac{1}{2}} \mid \phi> \\
& +\sum_{T^{\prime} t^{\prime \prime}} \sum_{a^{\prime}} \int d p^{\prime} p^{\prime 2} d q^{\prime} q^{\prime 2}<p q a^{>}\left|t_{1 c}^{T T^{\prime}}\right| p^{\prime} q^{\prime} a^{\prime}><p^{\prime} q^{\prime} a^{\prime}\left|P_{1 t^{\prime \prime}}^{T^{\prime}} G_{0}\right| T_{t^{\prime \prime}}^{T^{\prime}}>
\end{aligned}
$$


where $t_{1 c}^{T T^{\prime}}$ up to a factor is given by the screened pure Coulomb t-matrix $t_{p p}^{c R}$ contained in $t_{p p}^{1-1}$. Thus

$$
t_{1 c}^{T T^{\prime}}=f^{1 T 1 T^{\prime}} t_{p p}^{c R}
$$

and $f^{1 T 1 T^{\prime}}$ can be read of from (8) as $f^{1 \frac{1}{2} 1 \frac{1}{2}}=\frac{2}{3}=2 f^{1 \frac{3}{2} 1 \frac{3}{2}}, f^{1 \frac{1}{2} 1 \frac{3}{2}}=f^{1 \frac{3}{2} 1 \frac{1}{2}}=-\frac{\sqrt{2}}{3}$.

Inserting the factors $f^{1 T 1 T^{\prime}}$ it simply follows from (33) that

$$
<p q a^{>}\left|T_{1}^{\frac{1}{2}}>+\sqrt{2}<p q a^{>}\right| T_{1}^{\frac{3}{2}}>=0 .
$$

The physical meaning of that relation is trivially evident in the particle picture. One has

$$
\begin{aligned}
& \mid \gamma_{1}>=\frac{1}{\sqrt{6}}(|p p n>+| p n p>-2 \mid n p p>) \\
& \mid \gamma_{2}>=\frac{1}{\sqrt{3}}(|p p n>+| p n p>+\mid n p p>)
\end{aligned}
$$

where the sequence $p n p$ for instance corresponds to the sequence of particle numbers $1,2,3$.

Inserting the definitions (14) and (16) the relation (35) can be rewritten as

$$
<p q a^{>}\left|\left(<\gamma_{1}\left|+\sqrt{2}<\gamma_{2}\right|\right) T>=\sqrt{\frac{3}{2}}<p q a^{>}\right|(<p p n|+<p n p|) \mid T>.
$$

Now the $T$-amplitude projected on high angular momenta is proportional to the Coulomb $p p$ t-matrix, which acts in the 23-subsystem. Consequently the overlap in (38) is zero.

Now using (35) and (34) the relation (33) for $\left\langle p q a^{>} \mid T_{1}^{\frac{1}{2}}\right\rangle$ simplifies to

$$
\begin{aligned}
<p q a^{>} \mid T_{1}^{\frac{1}{2}}> & =\frac{1}{\sqrt{3}} \sum_{a^{\prime}} \int d p^{\prime} p^{\prime 2} d q^{\prime} q^{2}<p q a^{>}\left|t_{p p}^{c R}\right| p^{\prime} q^{\prime} a^{\prime}><p^{\prime} q^{\prime} a^{\prime}\left|P_{10}\right| \phi> \\
& +\frac{1}{\sqrt{3}} \sum_{a^{\prime}} \int d p^{\prime} p^{\prime 2} d q^{\prime} q^{\prime 2}<p q a^{>}\left|t_{p p}^{c R}\right| p^{\prime} q^{\prime} a^{\prime}><p^{\prime} q^{\prime} a^{\prime}\left|P_{10} G_{0}\right| T_{0}^{\frac{1}{2}}> \\
& -\frac{1}{3} \sum_{a^{\prime}} \int d p^{\prime} p^{\prime 2} d q^{\prime} q^{\prime 2} \sum_{a^{\prime \prime}} \int d p^{\prime \prime} p^{\prime \prime 2} d q^{\prime \prime} q^{\prime \prime 2}<p q a^{>}\left|t_{p p}^{c R}\right| p^{\prime} q^{\prime} a^{\prime}> \\
& <p^{\prime} q^{\prime} a^{\prime}\left|P_{11} G_{0}\right| p^{\prime \prime} q^{\prime \prime} a^{\prime \prime}<><p^{\prime \prime} q^{\prime \prime} a^{\prime \prime}<\left(\left|T_{1}^{\frac{1}{2}}>+\sqrt{2}\right| T_{1}^{\frac{3}{2}}>\right) .
\end{aligned}
$$

Because of (35) there are no contributions of the higher partial wave components and only the lower ones, denoted as $<p q a^{<} \mid$, are applied onto the amplitudes $\mid T>$. Due to (35) the same is true for $\left\langle p q a^{>}\right| T_{1}^{\frac{3}{2}}>$. For the sake of a simpler notation we introduce $|A>\equiv| p q a^{<}>$and $|B>\equiv| p q a^{>}>$in the following and drop the summation and integration signs.

Now we turn to the lower partial waves. From (27) - (29) and inserting $t_{t}^{T T^{\prime}}, P_{t t^{\prime}}^{T}$ explicitly we get

$$
<A\left|T_{0}^{1 / 2}>=-\frac{1}{2}<A\right| t_{n p}^{00} P_{00} \mid \phi>
$$




$$
\begin{aligned}
& -\frac{1}{2}<A\left|t_{n p}^{00} P_{00} G_{0}\right| A^{\prime}><A^{\prime} \mid T_{0}^{1 / 2}> \\
& -\frac{\sqrt{3}}{2}<A\left|t_{n p}^{00} P_{01} G_{0}\right| A^{\prime}><A^{\prime} \mid T_{1}^{1 / 2}> \\
& -\frac{\sqrt{3}}{2}<A\left|t_{n p}^{00} P_{01} G_{0}\right| B^{\prime}><B^{\prime} \mid T_{1}^{1 / 2}> \\
<A \mid T_{1}^{1 / 2}> & =\frac{1}{2 \sqrt{3}}<A\left|\left(t_{n p}^{10}+2 t_{p p}^{1-1}\right) P_{10}\right| \phi> \\
& +\frac{1}{2 \sqrt{3}}<A\left|\left(t_{n p}^{10}+2 t_{p p}^{1-1}\right) P_{10} G_{0}\right| A^{\prime}><A^{\prime} \mid T_{0}^{1 / 2}> \\
& -\frac{1}{6}<A\left|\left(t_{n p}^{10}+2 t_{p p}^{1-1}\right) P_{11} G_{0}\right| A^{\prime}><A^{\prime} \mid T_{1}^{1 / 2}> \\
& -\frac{1}{6}<A\left|\left(t_{n p}^{10}+2 t_{p p}^{1-1}\right) P_{11} G_{0}\right| B^{\prime}><B^{\prime} \mid T_{1}^{1 / 2}> \\
& +\frac{\sqrt{2}}{3}<A\left|\left(t_{n p}^{10}-t_{p p}^{1-1}\right) P_{11} G_{0}\right| A^{\prime}><A^{\prime} \mid T_{1}^{3 / 2}> \\
& +\frac{\sqrt{2}}{3}<A\left|\left(t_{n p}^{10}-t_{p p}^{1-1}\right) P_{11} G_{0}\right| B^{\prime}><B^{\prime} \mid T_{1}^{3 / 2}> \\
& =\frac{1}{\sqrt{6}}<A\left|\left(t_{n p}^{10}-t_{p p}^{1-1}\right) P_{10}\right| \phi> \\
& +\frac{1}{\sqrt{6}}<A\left|\left(t_{n p}^{10}-t_{p p}^{1-1}\right) P_{10} G_{0}\right| A^{\prime}><A^{\prime} \mid T_{0}^{1 / 2}> \\
& -\frac{1}{3 \sqrt{2}}<A\left|\left(t_{n p}^{10}-t_{p p}^{1-1}\right) P_{11} G_{0}\right| A^{\prime}><A^{\prime} \mid T_{1}^{1 / 2}> \\
& +\frac{1}{3}<A\left|\left(2 t_{n p}^{10}+t_{p p}^{1-1}\right) P_{11} G_{0}\right| A^{\prime}><A^{\prime} \mid T_{1}^{3 / 2}> \\
& -\frac{1}{3 \sqrt{2}}<A\left|\left(t_{n p}^{10}-t_{p p}^{1-1}\right) P_{11} G_{0}\right| B^{\prime}><B^{\prime} \mid T_{1}^{1 / 2}> \\
& +<A\left|\left(\frac{2}{3} t_{n p}^{10}+\frac{1}{3} t_{p p}^{1-1}\right) P_{11} G_{0}\right| B^{\prime}><B^{\prime} \mid T_{1}^{3 / 2}>
\end{aligned}
$$

Note $t_{p p}^{1-1}$ is the pp t-matrix driven by the strong and screened pp Coulomb force and is projected on a certain set of low partial waves.

In (41) and (42) one can use (35) to eliminate $<B^{\prime} \mid T_{1}^{3 / 2}>$ leading to

$$
\begin{aligned}
<A \mid T_{1}^{1 / 2}> & =\frac{1}{2 \sqrt{3}}<A\left|\left(t_{n p}^{10}+2 t_{p p}^{1-1}\right) P_{10}\right| \phi> \\
& +\frac{1}{2 \sqrt{3}}<A\left|\left(t_{n p}^{10}+2 t_{p p}^{1-1}\right) P_{10} G_{0}\right| A^{\prime}><A^{\prime} \mid T_{0}^{1 / 2}> \\
& -\frac{1}{6}<A\left|\left(t_{n p}^{10}+2 t_{p p}^{1-1}\right) P_{11} G_{0}\right| A^{\prime}><A^{\prime} \mid T_{1}^{1 / 2}> \\
& +\frac{\sqrt{2}}{3}<A\left|\left(t_{n p}^{10}-t_{p p}^{1-1}\right) P_{11} G_{0}\right| A^{\prime}><A^{\prime} \mid T_{1}^{3 / 2}> \\
& -\frac{1}{2}<A\left|t_{n p}^{10} P_{11} G_{0}\right| B^{\prime}><B^{\prime} \mid T_{1}^{1 / 2}>, \\
<A \mid T_{1}^{3 / 2}> & =\frac{1}{\sqrt{6}}<A\left|\left(t_{n p}^{10}-t_{p p}^{1-1}\right) P_{10}\right| \phi> \\
& +\frac{1}{\sqrt{6}}<A\left|\left(t_{n p}^{10}-t_{p p}^{1-1}\right) P_{10} G_{0}\right| A^{\prime}><A^{\prime} \mid T_{0}^{1 / 2}> \\
& -\frac{1}{2 \sqrt{2}}<A\left|\left(t_{n p}^{10}-t_{p p}^{1-1}\right) P_{11} G_{0}\right| A^{\prime}><A^{\prime} \mid T_{1}^{1 / 2}>
\end{aligned}
$$




$$
\begin{aligned}
& +\frac{1}{3}<A\left|\left(2 t_{n p}^{10}+t_{p p}^{1-1}\right) P_{11} G_{0}\right| A^{\prime}><A^{\prime} \mid T_{1}^{3 / 2}> \\
& -\frac{1}{\sqrt{2}}<A\left|t_{n p}^{10} P_{11} G_{0}\right| B^{\prime}><B^{\prime} \mid T_{1}^{1 / 2}>.
\end{aligned}
$$

The sum over the high partial waves acts on $\mid T_{1}^{1 / 2}>$ which, according to (39), is driven by the screened Coulomb t-matrix $t_{p p}^{c R}$. In order to avoid approximations we sum up the high partial waves to infinity and add and subtract the projection of $t_{p p}^{c R}$ on the finite number of low partial waves. Thus we put

$$
\left|B^{\prime}><B^{\prime}\right| T_{1}^{1 / 2}>\equiv \mid \tilde{T}_{1}^{1 / 2}>
$$

where according to (39)

$$
\begin{aligned}
\mid \tilde{T}_{1}^{1 / 2}> & =\frac{1}{\sqrt{3}} \tilde{t}_{p p}^{c R} P_{10}\left|\phi>+\frac{1}{\sqrt{3}} \tilde{t}_{p p}^{c R} P_{10} G_{0}\right| A^{\prime}><A^{\prime} \mid T_{0}^{\frac{1}{2}}> \\
& -\frac{1}{3} \tilde{t}_{p p}^{c R} P_{11} G_{0} \mid A^{\prime}><A^{\prime}\left(\left|T_{1}^{\frac{1}{2}}>+\sqrt{2}\right| T_{1}^{\frac{3}{2}}>\right)
\end{aligned}
$$

with

$$
\tilde{t}_{p p}^{c R} \equiv t_{p p}^{c R}-|A><A| t_{p p}^{c R}\left|A^{\prime}><A^{\prime}\right|
$$

In (46) the projection on partial waves to the right of $t_{p p}^{c R}$ has been carried out to infinite order leading to the 3 -dimensional screened Coulomb t-matrix $t_{p p}^{c R}$. But due to (47) also the partial wave projected $t_{p p}^{c R}$ matrix occurs. Now we insert (46) into the set (40), (43) and (44):

$$
\begin{aligned}
<A \mid T_{0}^{1 / 2}>= & -\frac{1}{2}<A\left|t_{n p}^{00} P_{00}\right| \phi> \\
& -\frac{1}{2}<A\left|t_{n p}^{00} P_{00} G_{0}\right| A^{\prime}><A^{\prime} \mid T_{0}^{1 / 2}> \\
& -\frac{\sqrt{3}}{2}<A\left|t_{n p}^{00} P_{01} G_{0}\right| A^{\prime}><A^{\prime} \mid T_{1}^{1 / 2}> \\
& -\frac{\sqrt{3}}{2}<A\left|t_{n p}^{00} P_{01} G_{0}\right| \tilde{T}_{1}^{1 / 2}>, \\
<A \mid T_{1}^{1 / 2}>= & \frac{1}{2 \sqrt{3}}<A\left|\left(t_{n p}^{10}+2 t_{p p}^{1-1}\right) P_{10}\right| \phi> \\
+ & \frac{1}{2 \sqrt{3}}<A\left|\left(t_{n p}^{10}+2 t_{p p}^{1-1}\right) P_{10} G_{0}\right| A^{\prime}><A^{\prime} \mid T_{0}^{1 / 2}> \\
- & \frac{1}{6}<A\left|\left(t_{n p}^{10}+2 t_{p p}^{1-1}\right) P_{11} G_{0}\right| A^{\prime}><A^{\prime} \mid T_{1}^{1 / 2}> \\
+ & \frac{\sqrt{2}}{3}<A\left|\left(t_{n p}^{10}-t_{p p}^{1-1}\right) P_{11} G_{0}\right| A^{\prime}><A^{\prime} \mid T_{1}^{3 / 2}> \\
- & \frac{1}{2}<A\left|t_{n p}^{10} P_{11} G_{0}\right| \tilde{T}_{1}^{1 / 2}>, \\
<A \mid T_{1}^{3 / 2}>= & \frac{1}{\sqrt{6}}<A\left|\left(t_{n p}^{10}-t_{p p}^{1-1}\right) P_{10}\right| \phi>
\end{aligned}
$$




$$
\begin{aligned}
& +\frac{1}{\sqrt{6}}<A\left|\left(t_{n p}^{10}-t_{p p}^{1-1}\right) P_{10} G_{0}\right| A^{\prime}><A^{\prime} \mid T_{0}^{1 / 2}> \\
& -\frac{1}{3 \sqrt{2}}<A\left|\left(t_{n p}^{10}-t_{p p}^{1-1}\right) P_{11} G_{0}\right| A^{\prime}><A^{\prime} \mid T_{1}^{1 / 2}> \\
& +\frac{1}{3}<A\left|\left(2 t_{n p}^{10}+t_{p p}^{1-1}\right) P_{11} G_{0}\right| A^{\prime}><A^{\prime} \mid T_{1}^{3 / 2}> \\
& -\frac{1}{\sqrt{2}}<A\left|t_{n p}^{10} P_{11} G_{0}\right| \tilde{T}_{1}^{1 / 2}>
\end{aligned}
$$

Eqs. (48) - (50) together with (46) form a closed set for amplitudes projected on low partial waves only. Since we anyhow iterate the equations and sum up the resulting series by Pade one can stay with that form. However, we prefer to eliminate (46) to arrive at our final form of coupled equations:

$$
\begin{aligned}
& <A\left|T_{0}^{1 / 2}>=-\frac{1}{2}<A\right| t_{n p}^{00} P_{00} \mid \phi> \\
& -\frac{1}{2}<A\left|t_{n p}^{00} P_{01} G_{0} \tilde{t}_{p p}^{c R} P_{10}\right| \phi> \\
& -\frac{1}{2}<A\left|t_{n p}^{00} P_{00} G_{0}\right| A^{\prime}><A^{\prime} \mid T_{0}^{1 / 2}> \\
& -\frac{1}{2}<A\left|t_{n p}^{00} P_{01} G_{0} \tilde{t}_{p p}^{c R} P_{10} G_{0}\right| A^{\prime}><A^{\prime} \mid T_{0}^{\frac{1}{2}}> \\
& -\frac{\sqrt{3}}{2}<A\left|t_{n p}^{00} P_{01} G_{0}\right| A^{\prime}><A^{\prime} \mid T_{1}^{1 / 2}> \\
& +\frac{1}{2 \sqrt{3}}<A\left|t_{n p}^{00} P_{01} G_{0} \tilde{t}_{p p}^{c R} P_{11} G_{0}\right| A^{\prime}><A^{\prime} \mid T_{1}^{\frac{1}{2}}> \\
& +\frac{1}{\sqrt{6}}<A\left|t_{n p}^{00} P_{01} G_{0} \tilde{t}_{p p}^{c R} P_{11} G_{0}\right| A^{\prime}><A^{\prime} \mid T_{1}^{\frac{3}{2}}>, \\
& <A\left|T_{1}^{1 / 2}>=\frac{1}{2 \sqrt{3}}<A\right|\left(t_{n p}^{10}+2 t_{p p}^{1-1}\right) P_{10} \mid \phi> \\
& -\frac{1}{2 \sqrt{3}}<A\left|t_{n p}^{10} P_{11} G_{0} \tilde{t}_{p p}^{c R} P_{10}\right| \phi> \\
& +\frac{1}{2 \sqrt{3}}<A\left|\left(t_{n p}^{10}+2 t_{p p}^{1-1}\right) P_{10} G_{0}\right| A^{\prime}><A^{\prime} \mid T_{0}^{1 / 2}> \\
& -\frac{1}{2 \sqrt{3}}<A\left|t_{n p}^{10} P_{11} G_{0} \tilde{t}_{p p}^{c R} P_{10} G_{0}\right| A^{\prime}><A^{\prime} \mid T_{0}^{\frac{1}{2}}> \\
& -\frac{1}{6}<A\left|\left(t_{n p}^{10}+2 t_{p p}^{1-1}\right) P_{11} G_{0}\right| A^{\prime}><A^{\prime} \mid T_{1}^{1 / 2}> \\
& +\frac{1}{6}<A\left|t_{n p}^{10} P_{11} G_{0} \tilde{t}_{p p}^{c R} P_{11} G_{0}\right| A^{\prime}><A^{\prime} \mid T_{1}^{\frac{1}{2}}> \\
& +\frac{\sqrt{2}}{3}<A\left|\left(t_{n p}^{10}-t_{p p}^{1-1}\right) P_{11} G_{0}\right| A^{\prime}><A^{\prime} \mid T_{1}^{3 / 2}> \\
& +\frac{\sqrt{2}}{6}<A\left|t_{n p}^{10} P_{11} G_{0} \tilde{t}_{p p}^{c R} P_{11} G_{0}\right| A^{\prime}><A^{\prime} \mid T_{1}^{\frac{3}{2}}>\text {, } \\
& <A\left|T_{1}^{3 / 2}>=\frac{1}{\sqrt{6}}<A\right|\left(t_{n p}^{10}-t_{p p}^{1-1}\right) P_{10} \mid \phi> \\
& -\frac{1}{\sqrt{6}}<A\left|t_{n p}^{10} P_{11} G_{0} \tilde{t}_{p p}^{c R} P_{10}\right| \phi>
\end{aligned}
$$




$$
\begin{aligned}
& +\frac{1}{\sqrt{6}}<A\left|\left(t_{n p}^{10}-t_{p p}^{1-1}\right) P_{10} G_{0}\right| A^{\prime}><A^{\prime} \mid T_{0}^{1 / 2}> \\
& -\frac{1}{\sqrt{6}}<A\left|t_{n p}^{10} P_{11} G_{0} \tilde{t}_{p p}^{c R} P_{10} G_{0}\right| A^{\prime}><A^{\prime} \mid T_{0}^{\frac{1}{2}}> \\
& -\frac{1}{3 \sqrt{2}}<A\left|\left(t_{n p}^{10}-t_{p p}^{1-1}\right) P_{11} G_{0}\right| A^{\prime}><A^{\prime} \mid T_{1}^{1 / 2}> \\
& +\frac{1}{3 \sqrt{2}}<A\left|t_{n p}^{10} P_{11} G_{0} \tilde{t}_{p p}^{c R} P_{11} G_{0}\right| A^{\prime}><A^{\prime} \mid T_{1}^{\frac{1}{2}}> \\
& +\frac{1}{3}<A\left|\left(2 t_{n p}^{10}+t_{p p}^{1-1}\right) P_{11} G_{0}\right| A^{\prime}><A^{\prime} \mid T_{1}^{3 / 2}> \\
& +\frac{1}{3}<A\left|t_{n p}^{10} P_{11} G_{0} \tilde{t}_{p p}^{c R} P_{11} G_{0}\right| A^{\prime}><A^{\prime} \mid T_{1}^{\frac{3}{2}}>
\end{aligned}
$$

Note that in all three equations there occur terms where the full 3-dimensional screened Coulomb t-matrix $t_{p p}^{c R}$ is sandwiched between permutation operators and occurs together with the strong np t-matrix. Due to (47) there are also other terms of that second order type in t-operators where, however, $t_{p p}^{c R}$ is projected onto the lower partial waves states.

Whereas all the other expressions are of our standard type [5] the ones with the 3-dimensional $t_{p p}^{c R}$ operator require special care and will be dealt with in appendices.

Now we would like to write down equations (51), (52) and (53) directly in our standard momentum space partial wave basis $\mid p q \alpha>$ which is an extension of $\mid p q a>$ by adding isospin quantum numbers

$$
|p q \alpha>\equiv| p q a>\left|\left(t \frac{1}{2}\right) T>=\right| p q(l s) j\left(\lambda \frac{1}{2}\right) I(j I) J\left(t \frac{1}{2}\right) T>.
$$

Though this appears as a repetition it is a necessity to cast the set into our standard form, which underlies our existing codes.

We distinguish between the partial wave states $\mid p q \alpha>$ with total $2 \mathrm{~N}$ angular momentum $j$ below some value $j_{\max }: j \leq j_{\max }$, in which the nuclear, $V_{N}$, as well as the pp screened Coulomb interaction, $V_{c}^{R}$ (in isospin $t=1$ states only), are acting, and the states $\mid p q \beta>$ with $j>j_{\max }$, for which only $V_{c}^{R}$ is acting in the pp subsystem. The states $\mid p q \alpha>$ and $\mid p q \beta>$ form a complete system of states

$$
\int p^{2} d p q^{2} d q\left(\sum_{\alpha}|p q \alpha\rangle\left\langle p q \alpha\left|+\sum_{\beta}\right| p q \beta\right\rangle\langle p q \beta|\right)=\mathrm{I} .
$$

Projecting Eq.(13) for $T \mid \Phi>$ on the $\mid p q \alpha>$ and $\mid p q \beta>$ states one gets the following system of coupled integral equations

$$
\begin{aligned}
\langle p q \alpha|T| \Phi\rangle & =\left\langle p q \alpha\left|t_{N+c}^{R} P\right| \Phi\right\rangle \\
& +\left\langle p q \alpha\left|t_{N+c}^{R} P G_{0} \sum_{\alpha^{\prime}} \int p^{2} d p^{\prime} q^{\prime 2} d q^{\prime}\right| p^{\prime} q^{\prime} \alpha^{\prime}\right\rangle\left\langle p^{\prime} q^{\prime} \alpha^{\prime}|T| \Phi\right\rangle
\end{aligned}
$$




$$
\begin{aligned}
& +\left\langle p q \alpha\left|t_{N+c}^{R} P G_{0} \sum_{\beta^{\prime}} \int p^{\prime 2} d p^{\prime} q^{\prime 2} d q^{\prime}\right| p^{\prime} q^{\prime} \beta^{\prime}\right\rangle\left\langle p^{\prime} q^{\prime} \beta^{\prime}|T| \Phi\right\rangle \\
\langle p q \beta|T| \Phi\rangle & =\left\langle p q \beta\left|t_{c}^{R} P\right| \Phi\right\rangle \\
& +\left\langle p q \beta\left|t_{c}^{R} P G_{0} \sum_{\alpha^{\prime}} \int p^{\prime 2} d p^{\prime} q^{\prime 2} d q^{\prime}\right| p^{\prime} q^{\prime} \alpha^{\prime}\right\rangle\left\langle p^{\prime} q^{\prime} \alpha^{\prime}|T| \Phi\right\rangle \\
& +\left\langle p q \beta\left|t_{c}^{R} P G_{0} \sum_{\beta^{\prime}} \int p^{\prime 2} d p^{\prime} q^{\prime 2} d q^{\prime}\right| p^{\prime} q^{\prime} \beta^{\prime}\right\rangle\left\langle p^{\prime} q^{\prime} \beta^{\prime}|T| \Phi\right\rangle
\end{aligned}
$$

where $t_{N+c}^{R}$ and $t_{c}^{R}$ are t-matrices generated by the interactions $V_{N}+V_{c}^{R}$ and $V_{c}^{R}$, respectively.

The third term on the right hand side of (57) is proportional to $<p q \beta\left|t_{c}^{R} P G_{0}\right| p^{\prime} q^{\prime} \beta^{\prime}><$ $p^{\prime} q^{\prime} \beta^{\prime} \mid t_{c}^{R}$. A direct calculation of its isospin part shows that independently from the value of the total isospin $T$ it vanishes. This corresponds to the result found in (35).

Inserting $<p q \beta|T| \Phi>$ from (57) into (56) one gets

$$
\begin{aligned}
\langle p q \alpha|T| \Phi\rangle= & \left\langle p q \alpha\left|t_{N+c}^{R} P\right| \Phi\right\rangle+\left\langle p q \alpha\left|t_{N+c}^{R} P G_{0} t_{c}^{R} P\right| \Phi\right\rangle \\
- & \left\langle p q \alpha\left|t_{N+c}^{R} P G_{0} \sum_{\alpha^{\prime}} \int p^{\prime 2} d p^{\prime} q^{\prime 2} d q^{\prime}\right| p^{\prime} q^{\prime} \alpha^{\prime}\right\rangle\left\langle p^{\prime} q^{\prime} \alpha^{\prime}\left|t_{c}^{R} P\right| \Phi\right\rangle \\
+ & \left\langle p q \alpha\left|t_{N+c}^{R} P G_{0} \sum_{\alpha^{\prime}} \int p^{\prime 2} d p^{\prime} q^{\prime 2} d q^{\prime}\right| p^{\prime} q^{\prime} \alpha^{\prime}\right\rangle\left\langle p^{\prime} q^{\prime} \alpha^{\prime}|T| \Phi\right\rangle \\
+ & \left\langle p q \alpha\left|t_{N+c}^{R} P G_{0} t_{c}^{R} P G_{0} \sum_{\alpha^{\prime}} \int p^{\prime 2} d p^{\prime} q^{\prime 2} d q^{\prime}\right| p^{\prime} q^{\prime} \alpha^{\prime}\right\rangle\left\langle p^{\prime} q^{\prime} \alpha^{\prime}|T| \Phi\right\rangle \\
- & \left\langle p q \alpha\left|t_{N+c}^{R} P G_{0} \sum_{\alpha^{\prime}} \int p^{\prime 2} d p^{\prime} q^{\prime 2} d q^{\prime}\right| p^{\prime} q^{\prime} \alpha^{\prime}\right\rangle\left\langle p^{\prime} q^{\prime} \alpha^{\prime}\right| t_{c}^{R} P G_{0} \\
& \sum_{\alpha^{\prime \prime}} \int p^{\prime \prime 2} d p^{\prime \prime} q^{\prime \prime 2} d q^{\prime \prime}\left|p^{\prime \prime} q^{\prime \prime} \alpha^{\prime \prime}\right\rangle\left\langle p^{\prime \prime} q^{\prime \prime} \alpha^{\prime \prime}|T| \Phi\right\rangle .
\end{aligned}
$$

We used again a relation corresponding to (47), now in isospin notation. This is a coupled set of integral equations in the space of only the states $|\alpha\rangle$, which incorporates the contributions of the pp Coulomb interaction from all partial wave states up to infinity. Using the definition of the $\mid \gamma>_{i}$ states from (10) a direct calculation shows that the set (58) is identical to the set (51), (52) and (53). It can be solved by iteration and Pade summation. There are two new leading terms $<p q \alpha\left|t_{N+c}^{R} P G_{0} t_{c}^{R} P\right| \Phi>$ and $-<p q \alpha\left|t_{N+c}^{R} P G_{0}\right| \alpha^{\prime}><\alpha^{\prime}\left|t_{c}^{R} P\right| \Phi>$. The first term must be calculated using directly the 3 -dimensional screened Coulomb t-matrix $t_{c}^{R}$, while the second term requires partial wave projected screened Coulomb t-matrix elements in the $|\alpha\rangle$ channels only. The kernel also contains two new terms. The term $<p q \alpha\left|t_{N+c}^{R} P G_{0} t_{c}^{R} P G_{0}\right| \alpha^{\prime}><\alpha^{\prime}|T| \Phi>$ must again be calculated with a 3 -dimensional screened Coulomb t-matrix while the second one, $-<p q \alpha\left|t_{N+c}^{R} P G_{0}\right| \alpha^{\prime}><\alpha^{\prime}\left|t_{c}^{R} P G_{0}\right| \alpha^{\prime \prime}><\alpha^{\prime \prime}|T| \Phi>$, involves only the partial wave projected screened Coulomb t-matrix elements in the $\mid \alpha>$ channels. The calculation of the new terms with the partial wave projected Coulomb t-matrices follows our standard procedure. Namely the two 
sub kernels $t_{N+c}^{R} P G_{0}$ and $t_{c}^{R} P G_{0}$ are applied consecutively on the corresponding state. The details how to calculate the new terms with the 3-dimensional screened Coulomb t-matrix are given in Appendix A.

Now we turn to the physical scattering amplitudes.

\section{THE ELASTIC PD ON-SHELL TRANSITION AMPLITUDE}

The transition amplitude for elastic scattering is given by [5, 18]

$$
\left\langle\Phi^{\prime}|U| \Phi\right\rangle=\left\langle\Phi^{\prime}\left|P G_{0}^{-1}+P T\right| \Phi\right\rangle
$$

The amplitude for elastic scattering given in (59) has two contributions. The first one is independent of the pp Coulomb force

$$
<\Phi^{\prime}\left|P G_{0}^{-1}\right| \Phi>=<\Phi^{\prime}|P V| \Phi>=-\frac{1}{2}<\phi^{\prime}\left|P_{00} V_{n p}^{00}\right| \phi>
$$

For the second one we use (17), (23) and (24) and decompose into lower and higher partial waves which yields using (46)

$$
\begin{aligned}
<\Phi^{\prime}|P T| \Phi> & =-\frac{1}{2}<\phi^{\prime}\left|P_{00}\right| T_{0}^{1 / 2}>-\frac{\sqrt{3}}{2}<\phi^{\prime}\left|P_{01}\right| T_{1}^{1 / 2}> \\
& =-\frac{1}{2}<\phi^{\prime}\left|P_{00}\right| A><A\left|T_{0}^{1 / 2}>-\frac{\sqrt{3}}{2}<\phi^{\prime}\right| P_{01}|A><A| T_{1}^{1 / 2}>-\frac{\sqrt{3}}{2}<\phi^{\prime}\left|P_{01}\right| \tilde{T}_{1}^{1 / 2}> \\
& =-\frac{1}{2}<\phi^{\prime}\left|P_{01} \tilde{t}_{p p}^{c R} P_{10}\right| \phi>-\frac{1}{2}<\phi^{\prime}\left|P_{00}\right| A><A \mid T_{0}^{1 / 2}> \\
& -\frac{1}{2}<\phi^{\prime}\left|P_{01} \tilde{t}_{p p}^{c R} P_{10} G_{0}\right| A><A\left|T_{0}^{1 / 2}>-\frac{\sqrt{3}}{2}<\phi^{\prime}\right| P_{01}|A><A| T_{1}^{1 / 2}> \\
& +\frac{1}{2 \sqrt{3}}<\phi^{\prime}\left|P_{01} \tilde{t}_{p p}^{c R} P_{11} G_{0}\right| A><A \mid\left(\left|T_{1}^{1 / 2}>+\sqrt{2}\right| T_{1}^{3 / 2}>\right) .
\end{aligned}
$$

Again besides standard low partial wave contributions terms occur with the 3-dimensional screened Coulomb t-matrix sandwiched between permutation operators.

To calculate the matrix element (59) one needs $\langle\vec{p} \vec{q}|T| \Phi\rangle$ composed of low and high partial wave contributions for $T \mid \Phi>$ (see (57)). Using the completeness relation (55) one gets:

$$
\begin{aligned}
& \langle\vec{p} \vec{q}|T| \Phi\rangle=\left\langle\vec{p} \vec{q}\left|\sum_{\alpha^{\prime}} \int p^{\prime 2} d p^{\prime} q^{\prime 2} d q^{\prime}\right| p^{\prime} q^{\prime} \alpha^{\prime}\right\rangle\left\langle p^{\prime} q^{\prime} \alpha^{\prime}|T| \Phi\right\rangle \\
& -\left\langle\vec{p} \vec{q}\left|\sum_{\alpha^{\prime}} \int p^{\prime 2} d p^{\prime} q^{\prime 2} d q^{\prime}\right| p^{\prime} q^{\prime} \alpha^{\prime}\right\rangle\left\langle p^{\prime} q^{\prime} \alpha^{\prime}\left|t_{c}^{R} P\right| \Phi\right\rangle \\
& -\left\langle\vec{p} \vec{q}\left|\sum_{\alpha^{\prime}} \int p^{\prime 2} d p^{\prime} q^{\prime 2} d q^{\prime}\right| p^{\prime} q^{\prime} \alpha^{\prime}\right\rangle\left\langle p^{\prime} q^{\prime} \alpha^{\prime}\left|t_{c}^{R} P G_{0} \sum_{\alpha^{\prime \prime}} \int p^{\prime \prime 2} d p^{\prime \prime} q^{\prime \prime 2} d q^{\prime \prime}\right| p^{\prime \prime} q^{\prime \prime} \alpha^{\prime \prime}\right\rangle\left\langle p^{\prime \prime} q^{\prime \prime} \alpha^{\prime \prime}|T| \Phi\right\rangle \\
& +\left\langle\vec{p} \vec{q}\left|t_{c}^{R} P\right| \Phi\right\rangle+\left\langle\vec{p} \vec{q}\left|t_{c}^{R} P G_{0} \sum_{\alpha^{\prime}} \int p^{\prime 2} d p^{\prime} q^{\prime 2} d q^{\prime}\right| p^{\prime} q^{\prime} \alpha^{\prime}\right\rangle\left\langle p^{\prime} q^{\prime} \alpha^{\prime}|T| \Phi\right\rangle .
\end{aligned}
$$


It follows, that in addition to the amplitudes $<p q \alpha|T| \Phi>$ also the partial wave projected amplitudes $<p q \alpha\left|t_{c}^{R} P\right| \Phi>$ and $<p q \alpha\left|t_{c}^{R} P G_{0}\right| \alpha^{\prime}><\alpha^{\prime}|T| \Phi>$ are required. The expressions for the contributions of these three terms to the transition amplitude for the elastic scattering (and the breakup reaction) are given in Appendix B. The last two terms in (62) must again be calculated using directly the 3-dimensional screened Coulomb t-matrices. In Appendix C] the expressions for $\left\langle\vec{p} \vec{q}\left|t_{c}^{R} P\right| \Phi\right\rangle$ (breakup) and $\left\langle\vec{p} \vec{q}\left|P t_{c}^{R} P\right| \Phi\right\rangle$ (elastic scattering) are given together with the expression for $<\Phi^{\prime}\left|P G_{0}^{-1}\right| \Phi>$. In Appendix $\mathrm{D}$ we describe how to get the last matrix element $<\vec{p} \vec{q}\left|t_{c}^{R} P G_{0}\right| \alpha^{\prime}><\alpha^{\prime}|T| \Phi>$.

\section{THE SCREENING LIMIT}

The set of coupled Faddeev equations (58) or (48)-(50) is well defined for a finite screening radius. It is an exact set assuming that the strong $\mathrm{NN}$ t-matrix can be neglected beyond a certain $j_{\max }$, which is justified. Further the pp screened Coulomb force is taken into account to infinite order in the partial wave decomposition in form of the 3 -dimensional screened Coulomb t-matrix $t_{p p}^{c R}$. The important challenge is to control the screening limit for the physical on shell amplitude (59). The contribution (60) is well defined and independent of the Coulomb force. The corresponding expression without partial-wave expansion is given in (C5).

In the second part given in (61) there are contributions without and with an explicit $\tilde{t}_{p p}^{c R}$ operator. The first term in (61) is responsible for the Rutherford scattering between proton and deuteron. It has the form

$$
<\phi^{\prime}\left|P_{01} \tilde{t}_{p p}^{c R} P_{10}\right| \phi>=<\phi^{\prime}\left|P_{01} t_{p p}^{c R} P_{10}\right| \phi>-<\phi^{\prime}\left|P_{01}\right| A><A\left|t_{p p}^{c R}\right| A^{\prime}><A^{\prime}\left|P_{10}\right| \phi>
$$

With $P_{01}=P_{10}=P_{12} P_{23}-P_{13} P_{23}$ and using simple symmetry properties one obtains

$$
<\phi^{\prime}\left|P_{01} t_{p p}^{c R} P_{10}\right| \phi>=2<\phi^{\prime}\left|P_{12} P_{23} t_{p p}^{c R}\left(P_{12} P_{23}-P_{13} P_{23}\right)\right| \phi>
$$

The 3-dimensional screened Coulomb t-matrix enters that matrix element totally off-shell, except for forward scattering. To show that it is sufficient to regard only the momentum space part of that matrix element. Using well known relations among Jacobi momenta and the symmetry property of the deuteron one obtains

$$
\begin{aligned}
& <\phi^{\prime}\left|P_{01} t_{p p}^{c R} P_{10}\right| \phi>\sim 2 \int d^{3} q^{\prime} \phi_{d}\left(1 / 2 \vec{q}_{0}{ }^{\prime}+\vec{q}^{\prime}\right) \phi_{d}\left(1 / 2 \vec{q}_{0}+\vec{q}^{\prime}\right) \\
& \left(t_{p p}^{c R}\left(-\vec{q}_{0}{ }^{\prime}-1 / 2 \vec{q}^{\prime}, 1 / 2 \vec{q}^{\prime}+\vec{q}_{0}, E\left(q^{\prime}\right)\right)-t_{p p}^{c R}\left(-\vec{q}_{0}{ }^{\prime}-1 / 2 \vec{q}^{\prime},-1 / 2 \vec{q}^{\prime}-\overrightarrow{q_{0}}, E\left(q^{\prime}\right)\right)\right.
\end{aligned}
$$


with

$$
E\left(q^{\prime}\right)=E-\frac{3}{4 m} q^{\prime 2}=E_{d}+\frac{3}{4 m} q_{0}^{2}-\frac{3}{4 m} q^{\prime 2} .
$$

It is easy to see that $\left(\vec{q}_{0}{ }^{\prime}+1 / 2 \vec{q}^{\prime}\right)^{2} / m \neq E\left(q^{\prime}\right) \neq\left(\vec{q}_{0}+1 / 2 \vec{q}^{\prime}\right)^{2} / m$ and obviously $\left(\vec{q}_{0}{ }^{\prime}+1 / 2 \vec{q}^{\prime}\right)^{2} / m \neq$ $\left(\vec{q}_{0}+1 / 2 \vec{q}^{\prime}\right)^{2} / m$ except for forward scattering. Now the off-shell screened Coulomb t-matrix converges for $R \rightarrow \infty$ towards the pure off-shell Coulomb t-matrix, which is well defined. Thus except for forward scattering that matrix element has a well defined screening limit.

It is interesting to regard the replacement $t_{p p}^{c R} \rightarrow V_{p p}^{c R} \equiv \frac{e^{2}}{r} e^{-r / R}$ which in the limit $R \rightarrow \infty$ turns (65) into

$$
\begin{aligned}
<\phi^{\prime}\left|P_{01} V_{p p}^{c R} P_{10}\right| \phi> & \sim \frac{-e^{2}}{\left(\vec{q}_{0}-\vec{q}_{0}^{\prime}\right)^{2}} \int d^{3} q^{\prime} \phi_{d}\left(1 / 2 \vec{q}_{0}{ }^{\prime}+\vec{q}^{\prime}\right) \phi_{d}\left(1 / 2 \vec{q}_{0}+\vec{q}^{\prime}\right) \\
& -\int d^{3} q^{\prime} \phi_{d}\left(1 / 2 \vec{q}_{0}{ }^{\prime}+\vec{q}^{\prime}\right) \phi_{d}\left(1 / 2 \vec{q}_{0}+\vec{q}^{\prime}\right) \frac{e^{2}}{\left(\vec{q}_{0}+\vec{q}_{0}{ }^{\prime}+\vec{q}^{\prime}\right)^{2}} .
\end{aligned}
$$

The first part is the Rutherford scattering amplitude multiplied by a deuteron form factor and the second term a correction due to antisymmetrisation of the two protons.

The partial wave projected piece in (63) has also a well defined screening limit due to the same reasons.

Next we regard the two matrix elements of $\tilde{t}_{p p}^{c R}$ in (61) integrated together with $\mid A>$-projected $T$-amplitudes. As before $t_{p p}^{c R}$ is off-shell from the left. On the right there is an intermediate momentum integration, which includes the on-shell point. In that case $t_{p p}^{c R}$ acquires an infinitely oscillating phase factor in the screening limit [19] which, however, is integrable. Thus it remains to verify that the free propagator singularity of $G_{0}$ does not coincide with that on-shell singularity of $t_{p p}^{c R}$. Inserting the momentum representation and working out the permutations the free propagator singularity is located at

$$
E_{d}+\frac{3}{4 m} q_{0}^{2}-\frac{\left(\vec{q}^{\prime}+1 / 2 \vec{q}\right)^{2}}{m}-\frac{3}{4 m} q^{2}=0
$$

where $\vec{q}^{\prime}$ and $\vec{q}$ are integration variables. On the other hand the singularity of $t_{p p}^{c R}\left(\vec{p}, \vec{p}^{\prime}, E\left(q^{\prime}\right)\right)$ arising for $\vec{p}-\vec{p}^{\prime}=0$ is located in the matrix element either at $\vec{q}_{0}{ }^{\prime}+\vec{q}^{\prime}+\vec{q}=0$ or at $\vec{q}_{0}{ }^{\prime}=\vec{q}$. This inserted into (68) yields in both cases a non vanishing expression. Thus in that respect all the three terms in (61) containing $t_{p p}^{c R}$ have a well defined screening limit. It remains to consider the partial wave projected T-amplitudes $\left\langle A \mid T_{t}^{T}\right\rangle$, which enter in the matrix elements in (61). They are the solutions of the coupled set of Faddeev equations (58) which are ill defined in the screening limit. However, we expect that in that limit these amplitudes $\left\langle A \mid T_{t}^{T}\right\rangle$ acquire only infinitely oscillating logarithmic phase factors, which can well be integrated over. In fact as will be 
demonstrated in a forthcoming paper only the on-shell amplitude $<A \mid T_{t}^{T}>$ with $p^{2}+\frac{3}{4} q^{2}=m E$, which appears in the breakup transition amplitude, acquires that oscillating factor.

Thus we come to the conjecture that the physical on-shell elastic pd amplitude has a well defined screening limit and does not require renormalisation. This might appear surprising at a first glance, since the on-shell pp scattering amplitude has not a well defined screening limit. Our explanation is that in the pd system the Coulomb force does not act between a proton and the center of mass of the deuteron but between the two protons, where one of them is part of the deuteron and therefore the pp Coulomb t-matrix is integrated over the deuteron wave function in the final state in the elastic scattering amplitude.

That analytical insight is well supported by our numerical results laid out in the following section.

The case of the pd breakup process is quite different and will be dealt with in a forthcoming study.

\section{NUMERICAL RESULTS}

To demonstrate the feasibility of our approach we applied the outlined formalism to a simple dynamical model in which the nucleon-nucleon force was restricted to act in ${ }^{1} S_{0}$ and ${ }^{3} S_{1}-{ }^{3} D_{1}$ partial waves only and taken from the CD Bonn potential [20]. The pp Coulomb force was screened exponentially

$$
V_{p p}^{R}(r)=\frac{e^{2}}{r} e^{-\left(\frac{r}{R}\right)^{n}}
$$

with the screening radius $R$ and $n=1$.

To investigate the screening limit $R \rightarrow \infty$ we generated a set of partial-wave decomposed tmatrices, $t_{c}^{R}$, based on the screened pp Coulomb force alone or for $t_{N+C}^{R}$ combined with the pp nuclear interaction, taking $R=20,40,60,80,100,120$ and $140 \mathrm{fm}$. With that dynamical input we solved the set of Faddeev equations (58) for the total angular momenta of the p-p-n system up to $J \leq \frac{15}{2}$ and both parities. In this first study we restricted ourselves to the perturbative approximation for the 3-dimensional pure Coulomb t-matrix: $t_{c}^{R}=V_{c}^{R}$. Of course in the future studies that approximation will be avoided and the full solution of the 3-dimensional LS equation for the screened pp Coulomb t-matrix will be used.

In Fig. 1 we show the convergence in the screening radius $R$ of the pd elastic scattering cross section and compare the pd and nd elastic scattering angular distributions at the incoming nucleon 
energy $E_{N}^{l a b}=13 \mathrm{MeV}$. On the scale of the figure the cross sections for $R=40-140 \mathrm{fm}$ are practically indistinguishable. The detailed picture of that convergence is depicted in Fig. 2, where the ratio of the cross sections obtained with the screening radius $R$ to those with $R=140 \mathrm{fm}$ is shown as a function of the c.m. scattering angle $\Theta_{c . m}$. It is clearly seen that already with the screening radius $R=40 \mathrm{fm}$ converged results for the cross section are achieved. Increasing further the value of $R$ provides cross sections which differ less than $\approx 1 \%$ up to the forward scattering angles $\Theta_{c . m .} \approx 10^{\circ}$. At very forward angles, where the pp Coulomb force is dominant, larger screening radii are required to get the cross section with the same precision.

The angular distributions shown in Figs. 1 and 2 were obtained taking in the elastic scattering transition amplitude (59) the exchange term $\left\langle\Phi^{\prime}\left|P G_{0}^{-1}\right| \Phi\right\rangle$ together with the first four terms in (62) contributing to $\left\langle\Phi^{\prime}|P T| \Phi\right\rangle$. In Fig. 3 we present how each term contributes to the cross section. When all terms are taken into account the resulting angular distribution is given by the solid line. The $\left\langle\Phi^{\prime}|P T| \Phi\right\rangle$ term ( dotted line related to the first term in (62) ) contributes significantly at all angles. At backward angles the largest contribution comes from the exchange term $\left\langle\Phi^{\prime}\left|P G_{0}^{-1}\right| \Phi\right\rangle$ (dashed line) while at forward angles the most important is the "Rutherford" term $\left\langle\Phi^{\prime}\left|P t_{c}^{R} P\right| \Phi\right\rangle$ (double-dashed-dotted line related to the fourth term in (62) calculated with the 3-dimensional screened Coulomb t-matrix $t_{c}^{R}$ (in this first study treated perturbatively as $t_{c}^{R}=V_{c}^{R}$ ). The two terms based on the partial-wave projected Coulomb t-matrix, $\left\langle\Phi^{\prime}\left|P t_{c}^{R} P\right| \Phi\right\rangle$ (dashed-double-dotted line related to the second term in (62) ) and $\left\langle\Phi^{\prime}\left|P t_{c}^{R} P G_{0} T\right| \Phi\right\rangle$ (dashed-dotted line related to the third term in (62) ), are about 2-orders of magnitude smaller and thus of minor importance. The fact that at very forward angles the contribution of the $\left\langle\Phi^{\prime}\left|P t_{c}^{R} P G_{0} T\right| \Phi\right\rangle$ is an order of magnitude smaller than the contribution of the $\left\langle\Phi^{\prime}\left|P t_{c}^{R} P\right| \Phi\right\rangle$ seems to justify the neglection of the last term $\left\langle\Phi^{\prime}\left|P t_{c}^{R} P G_{0} T\right| \Phi\right\rangle$ in (62) coming with the 3-dimensional screened Coulomb t-matrix. In future studies this term will be calculated to verify this statement.

In Fig. 4 we demonstrate numerically that the elastic pd amplitude has a well defined screening limit and does not require renormalization. The real and imaginary parts of the partial wave contribution $\left\langle\Phi^{\prime}\left|P\left(T-t_{c}^{R} P-t_{c}^{R} P G_{0} T\right)\right| \Phi\right\rangle$ to the elastic transition amplitude are shown for two combinations of the incoming and outgoing deuteron and proton spin projections and a number of screening radia $R=20,40,60,80,100,120$, and $140 \mathrm{fm}$. The additional term (60) is real and independent of the screening radius. The fourth term in (62) is also real under our approximation $t_{c}^{R}=V_{c}^{R}$ and for angles different from zero has a well defined screening limit. Moreover it is peaked in forward direction and would dominate terms shown. All lines are practically overlapping. That shows that not only the cross section but the pd elastic scattering amplitude itself does not develop 
an oscillating phase in the infinite screening limit.

\section{SUMMARY}

We developed and presented a novel approach to include the pp Coulomb force into the momentum space 3N Faddeev calculations. It is based on a standard formulation for short range forces and relies on a screening of the long-range Coulomb interaction. In order to avoid all uncertainties connected with an application of the partial wave expansion, unsuitable when working with longrange forces, we apply directly the 3-dimensional pp screened Coulomb t-matrix. Furthermore we strictly insisted to treat the pp Coulomb force in its proper coordinate.

Using a simple dynamical model for the nuclear part of the interaction we demonstrated the feasibility of that approach. We provided analytical arguments and showed numerically that the physical elastic pd scattering amplitude has a well defined screening limit and therefore does not require renormalisation. Well converged elastic pd cross sections have been achieved at finite screening radii. In this first study the 3-dimensional screened pure Coulomb t-matrix was replaced by the screened Coulomb potential and only a small number of partial wave states for the NN interaction was taken into account. This restriction will be removed in a forthcoming article.

\section{Acknowledgments}

This work was supported by the Polish 2008-2011 science funds as a research project No. N N202 077435. It was also partially supported by the Helmholtz Association through funds provided to the virtual institute "Spin and strong QCD"(VH-VI-231) and by the European CommunityResearch Infrastructure Integrating Activity "Study of Strongly Interacting Matter" (acronym HadronPhysics2, Grant Agreement n. 227431) under the Seventh Framework Programme of EU. H.W. would like to thank for the warm hospitality and support during the stay at TUNL, USA. The numerical calculations have been performed on the supercomputer cluster of the JSC, Jülich, Germany. 


\section{APPENDIX A: MATRIX ELEMENTS WITH 3-DIMENSIONAL SCREENED}

COULOMB T-MATRIX: $<p q \alpha\left|t_{N+c}^{R} P G_{0} t_{c}^{R} P G_{0}\right| \alpha^{\prime}><\alpha^{\prime}|T| \Phi>$ AND $<p q \alpha\left|t_{N+c}^{R} P G_{0} t_{c}^{R} P\right| \Phi>$

These terms must be calculated using directly the 3 -dimensional screened Coulomb t-matrix $t_{c}^{R}$. Introducing partial wave states $\mid p q \beta>$ in LS-coupling

$$
|p q \beta\rangle \equiv\left|p q(l \lambda) L\left(s \frac{1}{2}\right) S(L S) J\left(t \frac{1}{2}\right) T\right\rangle
$$

and using the recoupling

$$
|p q \alpha\rangle=\sum_{L S} \sqrt{\hat{j} \hat{I} \hat{L} \hat{L}}\left\{\begin{array}{ccc}
l & s & j \\
\lambda & \frac{1}{2} & I \\
L & S & J
\end{array}\right\}|p q \beta\rangle \equiv \sum_{\beta}\langle\beta \mid \alpha\rangle|p q \beta\rangle
$$

the matrix element $\left\langle p q \alpha\left|t_{N+c}^{R} P G_{0} t_{c}^{R} P G_{0}\right| \alpha^{\prime}\right\rangle\left\langle\alpha^{\prime}|T| \Phi\right\rangle$ can be written as

$$
\begin{aligned}
& \left\langle p q \alpha\left|t_{N+c}^{R} P G_{0} t_{c}^{R} P G_{0}\right| \alpha^{\prime}\right\rangle\left\langle\alpha^{\prime}|T| \Phi\right\rangle=\sum_{\tilde{\alpha}} \int \tilde{p}^{2} d \tilde{p} t_{\alpha \tilde{\alpha}}^{N+c, R}(p, \tilde{p} ; q) G_{0}(\tilde{p}, E(q)) \\
& \sum_{\tilde{\beta}}\langle\tilde{\alpha} \mid \tilde{\beta}\rangle \sum_{\alpha^{\prime}, \beta^{\prime}}\left\langle\beta^{\prime} \mid \alpha^{\prime}\right\rangle \sum_{\mu, \mu^{\prime}}\langle L \mu S M-\mu \mid J M\rangle\left\langle L^{\prime} \mu^{\prime} S^{\prime} M-\mu^{\prime} \mid J M\right\rangle \\
& \int p^{\prime 2} d p^{\prime} q^{\prime 2} d q^{\prime} G_{0}\left(p^{\prime}, q^{\prime}\right) Z\left(\tilde{p} q p^{\prime} q^{\prime}\right)
\end{aligned}
$$

where $E(q) \equiv E-\frac{3}{4 m} q^{2}, G_{0}(p, q) \equiv \frac{1}{E-\frac{p^{2}}{m}-\frac{3 q^{2}}{4 m}+i \epsilon}$ and

$$
\begin{aligned}
& Z\left(\tilde{p} q p^{\prime} q^{\prime}\right) \equiv \delta_{S S^{\prime}} \delta_{\mu \mu^{\prime}} \delta_{M_{T},-1 / 2} \delta_{M_{T^{\prime}},-1 / 2} 6 \sqrt{\hat{t} \hat{t}^{\prime}}\left\{\begin{array}{ccc}
1 / 2 & 1 / 2 & t \\
1 / 2 & T & 1
\end{array}\right\}\left\{\begin{array}{ccc}
1 / 2 & 1 / 2 & 1 \\
1 / 2 & T^{\prime} & t^{\prime}
\end{array}\right\} \\
& {\left[(-1)^{1+s+t^{\prime}} \sqrt{\hat{s} \hat{s}^{\prime}}\left\{\begin{array}{ccc}
1 / 2 & 1 / 2 & s \\
1 / 2 & S & s^{\prime}
\end{array}\right\} X_{1}\left(\tilde{p} q \tilde{\beta}, p^{\prime} q^{\prime} \beta^{\prime}\right)+\delta_{s s^{\prime}} X_{2}\left(\tilde{p} q \tilde{\beta}, p^{\prime} q^{\prime} \beta^{\prime}\right)\right] \text {. }}
\end{aligned}
$$

$X_{1}$ and $X_{2}$ are given by

$$
\begin{aligned}
& X_{1}\left(\tilde{p} q \tilde{\beta}, p^{\prime} q^{\prime} \beta^{\prime}\right)=\left\langle\tilde{p} q(\tilde{l} \lambda) L \mu\left|P_{12}^{m} P_{23}^{m} t_{c}^{R}\left(T, T^{\prime}\right) P_{12}^{m} P_{23}^{m}\right| p^{\prime} q^{\prime}\left(l^{\prime} \lambda^{\prime}\right) L^{\prime} \mu^{\prime}\right\rangle, \\
& X_{2}\left(\tilde{p} q \tilde{\beta}, p^{\prime} q^{\prime} \beta^{\prime}\right)=\left\langle\tilde{p} q(\tilde{l} \lambda) L \mu\left|P_{12}^{m} P_{23}^{m} t_{c}^{R}\left(T, T^{\prime}\right) P_{13}^{m} P_{23}^{m}\right| p^{\prime} q^{\prime}\left(l^{\prime} \lambda^{\prime}\right) L^{\prime} \mu^{\prime}\right\rangle,
\end{aligned}
$$

and the isospin matrix element of the 3 -dimensional screened Coulomb t-matrix is

$$
\left\langle\left(t \frac{1}{2}\right) T m_{T}\left|t_{c}^{R}\right|\left(t^{\prime} \frac{1}{2}\right) T^{\prime} m_{T^{\prime}}\right\rangle=\delta_{t 1} \delta_{t t^{\prime}} \delta_{M_{T} M_{T^{\prime}}} \delta_{M_{T^{\prime}},-\frac{1}{2}} t_{c}^{R}\left(T, T^{\prime}\right) .
$$


with

$$
\begin{array}{r}
t_{c}^{R}\left(T=\frac{1}{2}, T^{\prime}=\frac{1}{2}\right)=\frac{2}{3} t_{c}^{R} \\
t_{c}^{R}\left(T=\frac{1}{2}, T^{\prime}=\frac{3}{2}\right)=t_{c}^{R}\left(T=\frac{3}{2}, T^{\prime}=\frac{1}{2}\right)=-\frac{\sqrt{2}}{3} t_{c}^{R} \\
t_{c}^{R}\left(T=\frac{3}{2}, T^{\prime}=\frac{3}{2}\right)=\frac{1}{3} t_{c}^{R}
\end{array}
$$

The transposition operator $P_{i j}$ has been decomposed into three parts: $P_{i j}^{t}$ acting on isospin-, $P_{i j}^{s}$ on spin-, and $P_{i j}^{m}$ on momentum-components of the basis $\mid p q \alpha>$

$$
P=P_{12}^{m} P_{23}^{m} P_{12}^{s} P_{23}^{s} P_{12}^{t} P_{23}^{t}+P_{13}^{m} P_{23}^{m} P_{13}^{s} P_{23}^{s} P_{13}^{t} P_{23}^{t} .
$$

Because $X_{i}$ are scalars diagonal in $L$ and $\mu$ and in addition independent on $\mu$ one can write

$$
\begin{aligned}
X_{1} & =\left\langle\tilde{p} q(\tilde{l} \lambda) L \mu\left|P_{12}^{m} P_{23}^{m} t_{c}^{R}\left(T, T^{\prime}\right) P_{12}^{m} P_{23}^{m}\right| p^{\prime} q^{\prime}\left(l^{\prime} \lambda^{\prime}\right) L^{\prime} \mu^{\prime}\right\rangle \\
& =\frac{\delta_{L L^{\prime}}}{2 L+1} \sum_{\mu}\left\langle\tilde{p} q(\tilde{l} \lambda) L \mu\left|P_{12}^{m} P_{23}^{m} t_{c}^{R}\left(T, T^{\prime}\right) P_{12}^{m} P_{23}^{m}\right| p^{\prime} q^{\prime}\left(l^{\prime} \lambda^{\prime}\right) L \mu\right\rangle .
\end{aligned}
$$

Inserting in (A12) from the left and right a complete system of states $\mid \vec{p} \vec{q}>$ one gets

$$
\begin{aligned}
\mathrm{X}_{1}= & \int d p_{1} p_{1}^{2} \int d p_{1}{ }^{\prime} p_{1}^{2}{ }^{\prime} \int d q_{1} q_{1}^{2} \int d \hat{\tilde{p}} \int d \hat{q} \int d \hat{p}^{\prime} \int d \hat{q}^{\prime} \int d \hat{p}_{1} \int d \hat{p}_{1}{ }^{\prime} \int d \hat{q}_{1} \\
& \frac{\delta_{L L^{\prime}}}{2 L+1} \sum_{\mu} Y_{\tilde{l} \lambda}^{* L \mu}(\hat{\tilde{p}}, \hat{q}) t_{c}^{R}\left(T, T^{\prime} ; p_{1}, p_{1}{ }^{\prime}, \hat{p}_{1} \circ \hat{p}_{1}{ }^{\prime} ; E\left(q_{1}\right)\right) Y_{l^{\prime} \lambda^{\prime}}^{L \mu}\left(\hat{p}^{\prime}, \hat{q}^{\prime}\right) \\
& \delta\left(\overrightarrow{\tilde{p}}-\frac{1}{2} \vec{q}-\vec{q}_{1}\right) \delta\left(\vec{p}_{1}+\vec{q}+\frac{1}{2} \vec{q}_{1}\right) \delta\left(\vec{p}_{1}{ }^{\prime}-\frac{1}{2} \vec{q}_{1}-\vec{q}^{\prime}\right) \delta\left(\vec{p}^{\prime}+\vec{q}_{1}+\frac{1}{2} \vec{q}^{\prime}\right) .
\end{aligned}
$$

The quantity $\sum_{\mu} Y_{\tilde{l} \lambda}^{* L \mu}(\hat{\tilde{p}}, \hat{q}) Y_{l^{\prime} \lambda^{\prime}}^{L \mu}\left(\hat{p}^{\prime}, \hat{q}^{\prime}\right)$ is a scalar and therefore can only depend on scalars formed from $\hat{\tilde{p}}, \hat{q}, \hat{p}^{\prime}$, and $\hat{q}^{\prime}$. Only 5 of the possible scalars are independent e.q. $\hat{\tilde{p}} \circ \hat{q}, \hat{\tilde{p}} \circ \hat{p}^{\prime}, \hat{\tilde{p}} \circ \hat{p} q^{\prime}$, $\hat{q} \circ \hat{p}^{\prime}$, and $\hat{q} \circ \hat{q}^{\prime}$, and they determine the geometry of $\hat{\tilde{p}}, \hat{q}, \hat{p}^{\prime}$ and $\hat{q}^{\prime}$. Using the $\delta$-functions in (A13) it follows that

$$
\begin{aligned}
\overrightarrow{\tilde{p}} & =\frac{1}{2} \vec{q}+\vec{q}_{1} \\
\vec{p}_{1} & =-\vec{q}-\frac{1}{2} \vec{q}_{1} \\
\vec{p}_{1}^{\prime} & =\frac{1}{2} \vec{q}_{1}+\vec{q}^{\prime} \\
\vec{p}^{\prime} & =-\vec{q}_{1}-\frac{1}{2} \vec{q}^{\prime} .
\end{aligned}
$$

The above 5 scalar products can be expressed by the only independent scalar $\vec{p}_{1} \circ \vec{p}_{1}^{\prime}$ and by the magnitudes of seven vectors: $\overrightarrow{\tilde{p}}, \vec{q}, \vec{p}^{\prime}, \vec{q}^{\prime}, \vec{p}_{1}, \vec{q}_{1}$ and $\vec{p}_{1}{ }^{\prime}$. This together with (A14) enables to perform the integrations in (A13) by choosing the vector $\vec{q}_{1} \| \hat{z}$ and $\vec{q} \in(x-z)$. Such choice of coordinates makes that also $\overrightarrow{\tilde{p}}$ and $\vec{p}_{1} \in(x-z)$ and: $\phi_{\tilde{\mathrm{p}}}=0, \phi_{\mathrm{p}_{1}}=\pi, \phi_{\mathrm{p}_{1}^{\prime}}=\phi_{q^{\prime}}, \phi_{\mathrm{p}^{\prime}}=\phi_{q^{\prime}}+\pi$. 
As a result of the integration one gets

$$
\begin{aligned}
\mathrm{X}_{1}\left(\tilde{p} q \tilde{\beta}, p^{\prime} q^{\prime} \beta^{\prime}\right) & =\frac{32 \pi^{2}}{\tilde{p} p^{\prime} q q^{\prime}} \int_{q_{1}^{\min }}^{q_{1}^{\max }} d q_{1} \frac{\delta_{L L^{\prime}}}{2 L+1} \sum_{\mu} Y_{\tilde{l} \lambda}^{* L \mu}\left(\theta_{\tilde{p}}, \phi_{\tilde{p}}=0, \theta_{q}, \phi_{q}=0\right) \\
& \times \int_{0}^{2 \pi} d \phi_{q^{\prime}} t_{c}^{R}\left(T, T^{\prime} ; \sqrt{\tilde{p}^{2}+\frac{3}{4} q^{2}-\frac{3}{4} q_{1}^{2}}, \sqrt{p^{\prime 2}+\frac{3}{4} q^{\prime 2}-\frac{3}{4} q_{1}^{2}}, \hat{p}_{1} \circ \hat{p}_{1}{ }^{\prime}, E\left(q_{1}\right)\right) \\
& \times Y_{l^{\prime} \lambda^{\prime}}^{L \mu}\left(\theta_{p^{\prime}}, \phi_{p^{\prime}}=\phi_{q^{\prime}}+\pi, \theta_{q^{\prime}}, \phi_{q^{\prime}}\right)
\end{aligned}
$$

with $\hat{p}_{1} \circ \hat{p}_{1}^{\prime}=\cos \theta_{p_{1}} \cos \theta_{p_{1}^{\prime}}+\sin \theta_{p_{1}} \sin \theta_{p_{1}^{\prime}} \cos \phi_{q^{\prime}}$ and

$$
\begin{aligned}
\cos \theta_{p_{1}} & =\frac{\frac{1}{2} q_{1}^{2}+\frac{1}{4} q^{2}-\tilde{p}^{2}}{q_{1} \sqrt{\tilde{p}^{2}+\frac{3}{4} q^{2}-\frac{3}{4} q_{1}^{2}}} \\
\cos \theta_{p_{1}}, & =\frac{p^{\prime 2}-\frac{1}{2} q_{1}^{2}-\frac{1}{4} q^{\prime 2}}{q_{1} \sqrt{p^{\prime 2}+\frac{3}{4} q^{\prime 2}-\frac{3}{4} q_{1}^{2}}}
\end{aligned}
$$

In a similar way one gets for $X_{2}$

$$
\begin{aligned}
\mathrm{X}_{2}\left(\tilde{p} q \tilde{\beta}, p^{\prime} q^{\prime} \beta^{\prime}\right) & =\frac{32 \pi^{2}}{\tilde{p} p^{\prime} q q^{\prime}} \int_{q_{1}^{\min }}^{q_{1}^{\max }} d q_{1} \frac{(-1)^{l^{\prime}} \delta_{L L^{\prime}}}{2 L+1} \sum_{\mu} Y_{\tilde{l} \lambda}^{* L \mu}\left(\theta_{\tilde{p}}, \phi_{\tilde{p}}=0, \theta_{q}, \phi_{q}=0\right) \\
& \times \int_{0}^{2 \pi} d \phi_{q^{\prime}} t_{c}^{R}\left(T, T^{\prime} ; \sqrt{\tilde{p}^{2}+\frac{3}{4} q^{2}-\frac{3}{4} q_{1}^{2}}, \sqrt{p^{\prime 2}+\frac{3}{4} q^{\prime 2}-\frac{3}{4} q_{1}^{2}},-\hat{p}_{1} \circ \hat{p}_{1}{ }^{\prime}, E\left(q_{1}\right)\right) \\
& \times Y_{l^{\prime} \lambda^{\prime}}^{L \mu}\left(\theta_{p^{\prime}}, \phi_{p^{\prime}}=\phi_{q^{\prime}}+\pi, \theta_{q^{\prime}}, \phi_{q^{\prime}}\right)
\end{aligned}
$$

In (A15) and (A17) the limits of integrations over $q_{1}$ are

$$
\begin{aligned}
& q_{1}^{\min }=\max \left\{\left|\tilde{p}-\frac{q}{2}\right|,\left|p^{\prime}-\frac{q^{\prime}}{2}\right|\right\} \\
& q_{1}^{\max }=\min \left\{\tilde{p}+\frac{q}{2}, p^{\prime}+\frac{q^{\prime}}{2}\right\} .
\end{aligned}
$$

The limits (A18) restrict the region of $p^{\prime}$-values at given $\tilde{p}, q$ and $q^{\prime}$, for which $X_{1}$ and $X_{2}$ are nonzero. For $q^{\prime} \leq 2\left|\tilde{p}-\frac{q}{2}\right|$ this requires that $p^{\prime} \in\left(\left|\tilde{p}-\frac{q}{2}\right|-\frac{q^{\prime}}{2}, \tilde{p}+\frac{q}{2}+\frac{q^{\prime}}{2}\right)$, for $2\left|\tilde{p}-\frac{q}{2}\right|<q^{\prime} \leq 2(\tilde{p}+$ $\left.\frac{q}{2}\right): p^{\prime} \in\left(0, \tilde{p}+\frac{q}{2}+\frac{q^{\prime}}{2}\right)$, and for $q^{\prime}>2\left|\tilde{p}-\frac{q}{2}\right|$ and $q^{\prime}>2\left(\tilde{p}+\frac{q}{2}\right): p^{\prime} \in\left(\frac{q^{\prime}}{2}-\left(\tilde{p}+\frac{q}{2}\right), \frac{q^{\prime}}{2}+\tilde{p}+\frac{q}{2}\right)$. Inserting $X_{1}$ and $X_{2}$ from (A15) and (A17) into (A3) and (A4) and using the orthogonality of the CG-coefficients one gets finally

$$
\begin{aligned}
& \left\langle p q \alpha\left|t_{N+c}^{R} P G_{0} t_{c}^{R} P G_{0}\right| \alpha^{\prime}\right\rangle\left\langle\alpha^{\prime}|T| \Phi\right\rangle=\sum_{\tilde{\alpha}} \int \tilde{p}^{2} d \tilde{p} t_{\alpha \tilde{\alpha}}^{N+c, R}(p, \tilde{p} ; E(q)) G_{0}(\tilde{p}, q) \\
& \sum_{\tilde{\beta}}\langle\tilde{\alpha} \mid \tilde{\beta}\rangle \sum_{\alpha^{\prime}, \beta^{\prime}}\left\langle\beta^{\prime} \mid \alpha^{\prime}\right\rangle \delta_{S S^{\prime}} \delta_{\mu \mu^{\prime}} \delta_{M_{T},-1 / 2} \delta_{M_{T^{\prime}},-1 / 2} 6 \sqrt{\hat{t} \hat{t}^{\prime}}\left\{\begin{array}{ccc}
1 / 2 & 1 / 2 & t \\
1 / 2 & T & 1
\end{array}\right\}\left\{\begin{array}{ccc}
1 / 2 & 1 / 2 & 1 \\
1 / 2 & T^{\prime} & t^{\prime}
\end{array}\right\}
\end{aligned}
$$




$$
\int p^{\prime 2} d p^{\prime} q^{\prime 2} d q^{\prime}\left[(-1)^{1+s+t^{\prime}} \sqrt{\hat{s} \hat{s}^{\prime}}\left\{\begin{array}{ccc}
1 / 2 & 1 / 2 & s \\
1 / 2 & S & s^{\prime}
\end{array}\right\} X_{1}+\delta_{s s^{\prime}} X_{2}\right] G_{0}\left(p^{\prime}, q^{\prime}\right)<p^{\prime} q^{\prime} \alpha^{\prime}|T| \Phi>
$$

Performing the analogous steps and using

$$
\begin{aligned}
& \left\langle\mathrm{p}^{\prime} \mathrm{q}^{\prime} \alpha^{\prime} \mid \Phi\right\rangle=\frac{\delta\left(\mathrm{q}^{\prime}-\mathrm{q}_{0}\right)}{\mathrm{q}^{\prime 2}} \delta_{\mathrm{t} 0} \delta_{\mathrm{T} 1 / 2} \delta_{\mathrm{j} 1} \delta_{\mathrm{s} 1} \varphi_{l}\left(\mathrm{p}^{\prime}\right) \sqrt{\frac{2 \lambda+1}{4 \pi}}\left\langle 1 m_{d} I m_{n} \mid J m_{n}+m_{d}\right\rangle\left\langle\lambda 0 \frac{1}{2} m_{n} \mid I m_{n}\right\rangle \\
& \equiv \frac{\delta\left(\mathrm{q}^{\prime}-\mathrm{q}_{0}\right)}{\mathrm{q}^{\prime 2}} \delta_{\alpha^{\prime} \alpha_{0}} \varphi_{\alpha_{0}}\left(p^{\prime}\right) C_{\alpha_{0}},
\end{aligned}
$$

where it is assumed that the incoming nucleons momentum is parallel to the $\mathrm{z}$-axis, one gets the second matrix element $\left\langle p q \alpha\left|t_{N+c}^{R} P G_{0} t_{c}^{R} P\right| \Phi\right\rangle$ :

$$
\begin{aligned}
& \left\langle p q \alpha\left|t_{N+c}^{R} P G_{0} t_{c}^{R} P\right| \Phi\right\rangle=\sum_{\tilde{\alpha}} \int \tilde{p}^{2} d \tilde{p} t_{\alpha \tilde{\alpha}}^{N+c, R}(p, \tilde{p} ; E(q)) G_{0}(\tilde{p}, q) \\
& \sum_{\tilde{\beta}}\langle\tilde{\alpha} \mid \tilde{\beta}\rangle \sum_{\alpha_{0}, \beta_{0}}\left\langle\beta_{0} \mid \alpha_{0}\right\rangle C_{\alpha_{0}} \delta_{S S^{\prime}} \delta_{\mu \mu^{\prime}} \delta_{M_{T},-1 / 2} \delta_{M_{T^{\prime}},-1 / 2} 3 \sqrt{\hat{t} \hat{t}^{\prime}}\left\{\begin{array}{ccc}
1 / 2 & 1 / 2 & t \\
1 / 2 & T & 1
\end{array}\right\} \\
& \int p^{\prime 2} d p^{\prime} \varphi_{\alpha_{0}}\left(p^{\prime}\right)\left[(-)^{1+s} \sqrt{\hat{s} \hat{s}^{\prime}}\left\{\begin{array}{ccc}
1 / 2 & 1 / 2 & s \\
1 / 2 & S & s^{\prime}
\end{array}\right\} X_{1}\left(\tilde{p} q \tilde{\beta}, p^{\prime} q_{0} \beta_{0}\right)+\delta_{s s^{\prime}} X_{2}\left(\tilde{p} q \tilde{\beta}, p^{\prime} q_{0} \beta_{0}\right)\right]
\end{aligned}
$$

where $\left|\alpha_{0}>=\right|\left(l^{\prime} s^{\prime}\right) j^{\prime}\left(\lambda^{\prime} 1 / 2\right) I^{\prime}\left(j^{\prime} I^{\prime}\right) J\left(t^{\prime} 1 / 2\right) T^{\prime}=1 / 2>, l^{\prime}=0,2, t^{\prime}=0, T^{\prime}=\frac{1}{2}, s^{\prime}=j^{\prime}=1$ and

$$
\mathrm{C}_{\alpha_{0}}=\sqrt{\frac{2 \lambda^{\prime}+1}{4 \pi}}\left\langle 1 m_{d} I^{\prime} m_{n} \mid J m_{n}+m_{d}\right\rangle\left\langle\lambda^{\prime} 0 \frac{1}{2} m_{n} \mid I^{\prime} m_{n}\right\rangle \text {. }
$$

\section{APPENDIX B: PARTIAL WAVE PROJECTED TRANSITION MATRIX ELEMENTS:}

$<\vec{p} \vec{q}\left|\alpha^{\prime}><\alpha^{\prime}\right| T \mid \Phi>$ AND $<\vec{p} \vec{q}\left|\alpha^{\prime}><\alpha^{\prime}\right| P T \mid \Phi>$

Including spin $\left(m_{i}\right)$ and isospin $\left(\nu_{i}\right)$ projections of the nucleons one gets for the kinematically complete breakup configuration specified by Jacobi momenta $(\vec{p}, \vec{q})$ the following contribution to the transition matrix element:

$$
\begin{aligned}
& \left\langle\vec{p} q m_{1} m_{2} m_{3} \nu_{1} \nu_{2} \nu_{3}\left|\sum_{\alpha} \int p^{\prime 2} d p^{\prime} q^{\prime 2} d q^{\prime}\right| p^{\prime} q^{\prime} \alpha\right\rangle\left\langle p^{\prime} q^{\prime} \alpha|T| \Phi\right\rangle=
\end{aligned}
$$

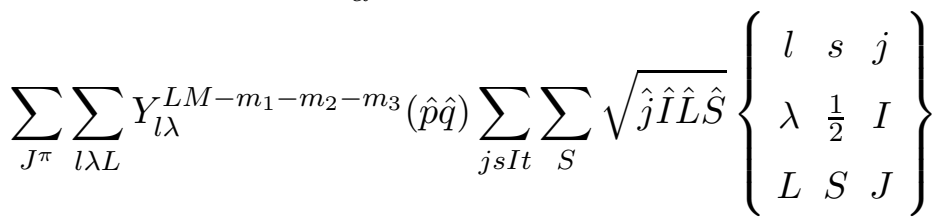

$$
\begin{aligned}
& \left\langle L S J, M-m_{1}-m_{2}-m_{3}, m_{1}+m_{2}+m_{3}\right\rangle\left\langle s \frac{1}{2} S, m_{2}+m_{3}, m_{1}\right\rangle \\
& \left\langle\frac{1}{2} \frac{1}{2} s, m_{2}, m_{3}\right\rangle\left\langle t \frac{1}{2} T, \nu_{2}+\nu_{3}, \nu_{1}\right\rangle\left\langle\frac{1}{2} \frac{1}{2} t, \nu_{2}, \nu_{3}\right\rangle\langle p q \alpha|T| \Phi\rangle
\end{aligned}
$$


where the incoming state $|\Phi\rangle \equiv\left|\vec{q}_{0}, \frac{1}{2} \mu\right\rangle\left|\varphi_{d}, 1 m_{d}\right\rangle$ is composed from the relative nucleon-deuteron motion with momentum $\vec{q}_{0} \| z$, the deuteron wave function $\varphi_{d}$, and $M=\mu+m_{d}$ is a sum of spin projections of the incoming nucleon and deuteron.

The corresponding contribution to the elastic scattering transition amplitude is:

$$
\begin{aligned}
& \left\langle\Phi^{\prime}\left|P \sum_{\alpha^{\prime}} \int p^{\prime 2} d p^{\prime} q^{\prime 2} d q^{\prime}\right| p^{\prime} q^{\prime} \alpha^{\prime}\right\rangle\left\langle p^{\prime} q^{\prime} \alpha^{\prime}|T| \Phi\right\rangle= \\
& \sum_{J^{\pi} M} \sum_{\alpha_{d}^{\prime} l_{0} \lambda_{0} I_{0}}\left\langle 1 I_{0} J, m_{d}^{\prime}, M-m_{d}^{\prime}\right\rangle\left\langle\lambda_{0} \frac{1}{2} I_{0}, M-m_{d}^{\prime}-\mu^{\prime}, \mu^{\prime}\right\rangle Y_{\lambda_{0}, M-m_{d}^{\prime}-\mu^{\prime}}\left(\hat{q}_{0}^{\prime}\right) \\
& \int q^{\prime 2} d q^{\prime} \int_{-1}^{1} d x \varphi_{l_{0}}\left(\pi_{1}\right) \frac{G_{\alpha_{0}, \alpha^{\prime}}\left(q_{0} q^{\prime} x\right)}{\pi_{1}^{l_{0}} \pi_{2}^{l_{\alpha_{d}^{\prime}}}}\left\langle\pi_{2} q^{\prime} \alpha^{\prime}|T| \Phi\right\rangle
\end{aligned}
$$

where $\left|\alpha_{d}>=\right|\left(l_{0} 1\right) 1\left(\lambda_{0} \frac{1}{2}\right) I_{0}\left(1 I_{0}\right) J,\left(\frac{1}{2} 0\right) T=\frac{1}{2}>, l_{0}=0,2, \varphi_{0,2}$ is the deuteron wave function, and $\pi_{1}=\sqrt{q^{\prime 2}+\frac{1}{4} q_{0}^{2}+q^{\prime} q_{0} x}, \pi_{2}=\sqrt{q_{0}^{2}+\frac{1}{4} q^{\prime 2}+q^{\prime} q_{0} x}$.

APPENDIX C: $\left\langle\Phi^{\prime}\left|P G_{0}^{-1}\right| \Phi\right\rangle$ AND MATRIX ELEMENTS WITH 3-DIMENSIONAL COULOMB T-MATRIX: $\left\langle\vec{p} \vec{q} m_{i} \nu_{i}\left|t_{c}^{R} P\right| \Phi\right\rangle$ AND $\left\langle\Phi^{\prime}\left|P t_{c}^{R} P\right| \Phi\right\rangle$

Using

$$
\begin{aligned}
& P\left|\vec{p} \vec{q} m_{1} m_{2} m_{3} \nu_{1} \nu_{2} \nu_{3}\right\rangle=\left|-\frac{1}{2} \vec{p}+\frac{3}{4} \vec{q},-\vec{p}-\frac{1}{2} \vec{q}, m_{3} m_{1} m_{2} \nu_{3} \nu_{1} \nu_{2}\right\rangle \\
& +\left|-\frac{1}{2} \vec{p}-\frac{3}{4} \vec{q}, \vec{p}-\frac{1}{2} \vec{q}, m_{2} m_{3} m_{1} \nu_{2} \nu_{3} \nu_{1}\right\rangle
\end{aligned}
$$

and

$$
\begin{aligned}
& \left\langle\vec{p} \vec{q} m_{1} m_{2} m_{3} \nu_{1} \nu_{2} \nu_{3} \mid \Phi\right\rangle \equiv\left\langle\vec{p} \vec{q} m_{1} m_{2} m_{3} \nu_{1} \nu_{2} \nu_{3} \mid \varphi_{d} 1 m_{d} t=00 \frac{1}{2} m_{N} \frac{1}{2} m_{t} \overrightarrow{q_{0}}\right\rangle= \\
& \sum_{L=0,2} \varphi_{L}(p) Y_{L, M=m_{d}-m_{2}-m_{3}}(\hat{p})\left\langle L 11, M, m_{d}-M\right\rangle \\
& \left\langle\frac{1}{2} \frac{1}{2} 1, m_{2} m_{3} m_{d}-M\right\rangle\left\langle\frac{1}{2} \frac{1}{2} 0, \nu_{2} \nu_{3} 0\right\rangle \delta_{m_{1} m_{N}} \delta_{\nu_{1} m_{t}} \delta\left(\vec{q}-\vec{q}_{0}\right)
\end{aligned}
$$

one gets the following expression for the contribution of the matrix element of the $t_{c}^{R} P$ to the breakup transition amplitude

$$
\begin{aligned}
& \left\langle\vec{p} \vec{q} m_{i} \nu_{i}\left|t_{c}^{R} P\right| \Phi\right\rangle= \\
& \delta_{m_{3}, m_{N}} \delta_{\nu_{3}, m_{t_{N}}} \delta_{\nu_{3},+\frac{1}{2}} \delta_{\nu_{2},+\frac{1}{2}} \sum_{L} \varphi_{L}\left(\left|\vec{q}+\frac{1}{2} \vec{q}_{0}\right|\right) Y_{L, m_{d}-m_{1}-m_{2}}\left(\vec{q}+\frac{1}{2} \vec{q}_{0}\right) \\
& \left(L 11, m_{d}-m_{1}-m_{2}, m_{1}+m_{2}\right) \\
& \times\left(\frac{1}{2} \frac{1}{2} 1, m_{1} m_{2}\right) \frac{\sqrt{2}}{2}(-)^{\frac{1}{2}-\nu_{1}} \delta_{\nu_{1},-\nu_{2}}\left\langle\vec{p}\left|t_{c}^{R}\left(E-\frac{3}{4 m} q^{2}\right)\right|-\frac{1}{2} \vec{q}-\vec{q}_{0}\right\rangle+
\end{aligned}
$$




$$
\begin{aligned}
& \delta_{m_{2}, m_{N}} \delta_{\nu_{2}, m_{t_{N}}} \delta_{\nu_{2},+\frac{1}{2}} \delta_{\nu_{3},+\frac{1}{2}} \sum_{L} \varphi_{L}\left(\left|\vec{q}+\frac{1}{2} \vec{q}_{0}\right|\right) Y_{L, m_{d}-m_{3}-m_{1}}\left(-\vec{q}-\hat{\frac{1}{2}} \vec{q}_{0}\right) \\
& \left(L 11, m_{d}-m_{3}-m_{1}, m_{3}+m_{1}\right) \\
& \times\left(\frac{1}{2} \frac{1}{2} 1, m_{3} m_{1}\right) \frac{\sqrt{2}}{2}(-)^{\frac{1}{2}-\nu_{3}} \delta_{\nu_{3},-\nu_{1}}\left\langle\vec{p}\left|t_{c}^{R}\left(E-\frac{3}{4 m} q^{2}\right)\right| \frac{1}{2} \vec{q}+\vec{q}_{0}\right\rangle .
\end{aligned}
$$

The corresponding contribution to the elastic scattering transition amplitude from $P t_{c}^{R} P$ is

$$
\begin{aligned}
& \left\langle\Phi^{\prime}\left|P t_{c}^{R} P\right| \Phi\right\rangle= \\
& \sum_{m_{1} m_{2}} \sum_{L, \bar{L}} \int d \vec{q} \varphi_{L}\left(\left|\vec{q}+\frac{1}{2} \vec{q}_{0}^{\prime}\right|\right) Y_{L, m_{d^{\prime}}-m_{1}-m_{2}}^{*}\left(\vec{q}+\frac{\hat{1}}{2} \vec{q}_{0}^{\prime}\right) \varphi_{\bar{L}}\left(\left|\vec{q}+\frac{1}{2} \vec{q}_{0}\right|\right) Y_{\bar{L}, m_{d}-m_{1}-m_{2}}\left(\vec{q}+{ }^{\hat{q}} \vec{q}_{0}\right) \\
& \left(L 11 \mid m_{d^{\prime}}-m_{1}-m_{2}, m_{1}+m_{2}, m_{d}^{\prime}\right)\left(\frac{1}{2} m_{1} \frac{1}{2} m_{2} \mid 1 m_{1}+m_{2}\right) \\
& \left(\bar{L} 11 \mid m_{d}-m_{1}-m_{2}, m_{1}+m_{2}, m_{d}\right)\left(\frac{1}{2} m_{1} \frac{1}{2} m_{2} \mid 1 m_{1}+m_{2}\right) \delta_{m_{N^{\prime}}, m_{N}} \delta_{m_{N_{N^{\prime}}}, m_{t_{N}}} \\
& t_{c}^{R}\left(\left|\frac{1}{2} \vec{q}+\vec{q}_{0}^{\prime}\right|,\left|\frac{1}{2} \vec{q}+\vec{q}_{0}\right|, \cos \theta=\frac{\frac{1}{4} q^{2}+\vec{q}_{0} \cdot \vec{q}_{0}^{\prime}+\frac{1}{2} \vec{q} \cdot\left(\vec{q}_{0}+\vec{q}_{0}^{\prime}\right)}{\left|\frac{1}{2} \vec{q}+\vec{q}_{0}^{\prime}\right|\left|\frac{1}{2} \vec{q}+\vec{q}_{0}\right|} ; E-\frac{3}{4 m} q^{2}\right) \\
& -\sum_{m_{1}} \sum_{L, \bar{L}} \int d \vec{q} \varphi_{L}\left(\left|\vec{q}+\frac{1}{2} \vec{q}_{0}^{\prime}\right|\right) Y_{L, m_{d^{\prime}}-m_{1}-m_{N}}^{*}\left(\vec{q}+\frac{\hat{1}}{2} \vec{q}_{0}^{\prime}\right) \varphi_{\bar{L}}\left(\left|\vec{q}+\frac{1}{2} \vec{q}_{0}\right|\right) Y_{\bar{L}, m_{d}-m_{N^{\prime}}-m_{1}}\left(\vec{q}+\frac{\hat{1}}{2} \vec{q}_{0}\right) \\
& \left(L 11 \mid m_{d^{\prime}}-m_{1}-m_{N}, m_{1}+m_{N}, m_{d}^{\prime}\right)\left(\frac{1}{2} m_{1} \frac{1}{2} m_{N} \mid 1 m_{1}+m_{N}\right) \\
& \left(\bar{L} 11 \mid m_{d}-m_{N^{\prime}}-m_{1}, m_{N^{\prime}}+m_{1}, m_{d}\right)\left(\frac{1}{2} m_{N^{\prime}} \frac{1}{2} m_{1} \mid 1 m_{N^{\prime}}+m_{1}\right) \delta_{m_{t_{N}}, m_{t} N_{N^{\prime}}} \\
& t_{c}^{R}\left(\left|\frac{1}{2} \vec{q}+\vec{q}_{0}^{\prime}\right|,\left|\frac{1}{2} \vec{q}+\vec{q}_{0}\right|, \cos \theta=\frac{\frac{1}{4} q^{2}+\vec{q}_{0} \cdot \vec{q}_{0}^{\prime}+\frac{1}{2} \vec{q} \cdot\left(\vec{q}_{0}+\vec{q}_{0}^{\prime}\right)}{\left|\frac{1}{2} \vec{q}+\vec{q}_{0}^{\prime}\right|\left|\frac{1}{2} \vec{q}+\vec{q}_{0}\right|} ; E-\frac{3}{4 m} q^{2}\right)
\end{aligned}
$$

where $\left\langle\vec{p}\left|t_{c}^{R}\right| \vec{p}^{\prime}\right\rangle \equiv t_{c}^{R}\left(|\vec{p}|,\left|\vec{p}^{\prime}\right|, \hat{p} \cdot \hat{p}^{\prime}\right)$.

The matrix element $<\Phi^{\prime}\left|P G_{0}^{-1}\right| \Phi>$ is given by

$$
\begin{aligned}
& \left\langle\Phi^{\prime}\left|P G_{0}^{-1}\right| \Phi\right\rangle=-\left[\frac{q_{0}^{2}}{m_{p}}\left(\frac{5}{4}+\cos \theta\right)+\left|E_{d}\right|\right] \sum_{L L^{\prime} m_{2}} \varphi_{L^{\prime}}\left(\left|\vec{q}_{0}+\frac{1}{2} \vec{q}_{0}{ }^{\prime}\right|\right) \\
& Y_{L^{\prime}, M^{\prime}=m_{d}^{\prime}-m_{N}-m_{2}}\left(\vec{q}_{0}+\frac{\hat{1}}{2} \vec{q}_{0}{ }^{\prime}\right)\left\langle L^{\prime} 11, M^{\prime}, m_{d}^{\prime}-M^{\prime}\right\rangle\left\langle\frac{1}{2} \frac{1}{2} 1, m_{N} m_{2} m_{d}^{\prime}-M^{\prime}\right\rangle \\
& \varphi_{L}\left(\left|\frac{1}{2} \vec{q}_{0}+\vec{q}_{0}^{\prime}\right|\right) Y_{L, M=m_{d}-m_{2}-m_{N}}\left(\frac{1}{2} \vec{q}_{0}+\vec{q}_{0}{ }^{\prime}\right)\left\langle L 11, M, m_{d}-M\right\rangle\left\langle\frac{1}{2} \frac{1}{2} 1, m_{2} m_{N} m_{d}-M\right\rangle
\end{aligned}
$$

\section{APPENDIX D: MATRIX ELEMENTS WITH 3-DIMENSIONAL COULOMB T-} MATRIX: $<\vec{p} \vec{q} m_{i} \nu_{i}\left|t_{c}^{R} P G_{0}\right| \alpha^{\prime}><\alpha^{\prime}|T| \Phi>$ AND $<\Phi^{\prime}\left|P t_{c}^{R} P G_{0}\right| \alpha^{\prime}><\alpha^{\prime}|T| \Phi>$

Inserting two complete set of states $\mid \vec{p} \vec{q} m_{i} \nu_{i}>\left(m_{i}\right.$ and $\nu_{i}$ are nucleons spin- and isospinprojections) and $\mid p q \alpha>$ one gets

$$
\begin{aligned}
\left\langle\vec{p} \vec{q} m_{i} \nu_{i}\left|t_{c}^{R} P G_{0} T\right| \Phi\right\rangle & =\int d \vec{p}^{\prime} t_{c}^{R}\left(\vec{p}, \vec{p}^{\prime} ; E(q)\right) \sum_{\alpha} \int d p^{\prime \prime} p^{\prime \prime 2} d q^{\prime \prime} q^{\prime \prime 2}\left\langle\vec{p}^{\prime} \vec{q} m_{i} \nu_{i}|P| p^{\prime \prime} q^{\prime \prime} \alpha\right\rangle \\
& \times G_{0}\left(p^{\prime \prime}, q^{\prime \prime}\right)\left\langle p^{\prime \prime} q^{\prime \prime} \alpha|T| \Phi\right\rangle .
\end{aligned}
$$


The permutation matrix element $\left\langle\vec{p}^{\prime} \vec{q} m_{i} \nu_{i}|P| p^{\prime \prime} q^{\prime \prime} \alpha\right\rangle$ is given by

$$
\begin{aligned}
& \left\langle\vec{p}^{\prime} \vec{q} m_{i} \nu_{i}|P| p^{\prime \prime} q^{\prime \prime} \alpha\right\rangle=\sum_{m_{i}^{\prime}, \nu_{i}^{\prime}} \int d \vec{p}_{4} d \vec{q}_{4}\left\langle\vec{p}^{\prime} \vec{q} m_{i} \nu_{i}|P| \vec{p}_{4} \vec{q}_{4} m_{i}^{\prime} \nu_{i}^{\prime}\right\rangle\left\langle\vec{p}_{4} \vec{q}_{4} m_{i}^{\prime} \nu_{i}^{\prime} \mid p^{\prime \prime} q^{\prime \prime} \alpha\right\rangle= \\
& \sum_{m_{i}^{\prime}, \nu_{i}^{\prime}} \int d \vec{p}_{4} d \overrightarrow{q_{4}}\left[\delta\left(\vec{p}^{\prime}-\overrightarrow{\pi_{1}}\right) \delta\left(\vec{p}_{4}+\overrightarrow{\pi_{2}}\right) \delta_{m_{1} m_{3}^{\prime}} \delta_{m_{2} m_{1}^{\prime}} \delta_{m_{3} m_{2}^{\prime}} \delta_{\nu_{1} \nu_{3}^{\prime}} \delta_{\nu_{2} \nu_{1}^{\prime}} \delta_{\nu_{3} \nu_{2}^{\prime}}\right. \\
& \left.+\delta\left(\vec{p}^{\prime}+\overrightarrow{\pi_{1}}\right) \delta\left(\vec{p}_{4}-\overrightarrow{\pi_{2}}\right) \delta_{m_{1} m_{2}^{\prime}} \delta_{m_{2} m_{3}^{\prime}} \delta_{m_{3} m_{1}^{\prime}} \delta_{\nu_{1} \nu_{2}^{\prime}} \delta_{\nu_{2} \nu_{3}^{\prime}} \delta_{\nu_{3} \nu_{1}^{\prime}}\right] \\
& \sum_{L S} \sqrt{\hat{j} \hat{I} \hat{L} \hat{S}}\left\{\begin{array}{ccc}
l & s & j \\
\lambda & \frac{1}{2} & I \\
L & S & J
\end{array}\right\} \sum_{M_{L}}\left\langle\frac{1}{2} m_{2}^{\prime} \frac{1}{2} m_{3}^{\prime} \mid s m_{2}^{\prime}+m_{3}^{\prime}\right\rangle\left\langle s m_{2}^{\prime}+m_{3}^{\prime} \frac{1}{2} m_{1}^{\prime} \mid S m_{1}^{\prime}+m_{2}^{\prime}+m_{3}^{\prime}\right\rangle \\
& \left\langle\frac{1}{2} \nu_{2}^{\prime} \frac{1}{2} \nu_{3}^{\prime} \mid t \nu_{2}^{\prime}+\nu_{3}^{\prime}\right\rangle\left\langle t \nu_{2}^{\prime}+\nu_{3}^{\prime} \frac{1}{2} \nu_{1}^{\prime} \mid T \nu_{1}^{\prime}+\nu_{2}^{\prime}+\nu_{3}^{\prime}\right\rangle\left\langle L M_{L} S m_{1}^{\prime}+m_{2}^{\prime}+m_{3}^{\prime} \mid J m_{p}+m_{d}\right\rangle \\
& \frac{\delta\left(p^{\prime \prime}-p_{4}\right)}{p^{\prime \prime 2}} \frac{\delta\left(q^{\prime \prime}-q_{4}\right)}{q^{\prime \prime 2}} Y_{l \lambda}^{L M_{L}}\left(\hat{p}_{4}, \hat{q}_{4}\right)
\end{aligned}
$$

where

$$
\begin{aligned}
& \overrightarrow{\pi_{1}}=\frac{1}{2} \vec{q}+\vec{q}_{4} \\
& \overrightarrow{\pi_{2}}=\vec{q}+\frac{1}{2} \overrightarrow{q_{4}} .
\end{aligned}
$$

Performing in ( $(\overline{\mathrm{D} 2})$ integrations over $p^{\prime \prime}$ and $q^{\prime \prime}$ leads to

$$
\begin{aligned}
& \left\langle\vec{p} \vec{q} m_{1} m_{2} m_{3} \nu_{1} \nu_{2} \nu_{3}\left|t_{c}^{R} P G_{0} T\right| \Phi\right\rangle=\int d \vec{p}^{\prime} d \vec{p}_{4} d \vec{q}_{4} t_{c}^{R}\left(\vec{p}, \vec{p}^{\prime} ; E(q)\right) \\
& \times \sum_{\alpha m_{i}^{\prime} \nu_{i}^{\prime}}\left[\delta\left(\vec{p}^{\prime}-\overrightarrow{\pi_{1}}\right) \delta\left(\vec{p}_{4}+\overrightarrow{\pi_{2}}\right) \delta_{m_{1} m_{3}^{\prime}} \delta_{m_{2} m_{1}^{\prime}} \delta_{m_{3} m_{2}^{\prime}} \delta_{\nu_{1} \nu_{3}^{\prime}} \delta_{\nu_{2} \nu_{1}^{\prime}} \delta_{\nu_{3} \nu_{2}^{\prime}}\right. \\
& \left.+\delta\left(\vec{p}^{\prime}+\overrightarrow{\pi_{1}}\right) \delta\left(\vec{p}_{4}-\overrightarrow{\pi_{2}}\right) \delta_{m_{1} m_{2}^{\prime}} \delta_{m_{2} m_{3}^{\prime}} \delta_{m_{3} m_{1}^{\prime}} \delta_{\nu_{1} \nu_{2}^{\prime}} \delta_{\nu_{2} \nu_{3}^{\prime}} \delta_{\nu_{3} \nu_{1}^{\prime}}\right] \\
& \sum_{L S} \sqrt{\hat{j} \hat{I} \hat{L} \hat{S}}\left\{\begin{array}{ccc}
l & s & j \\
\lambda & \frac{1}{2} & I \\
L & S & J
\end{array}\right\} \sum_{M_{L}}\left\langle\frac{1}{2} m_{2}^{\prime} \frac{1}{2} m_{3}^{\prime} \mid s m_{2}^{\prime}+m_{3}^{\prime}\right\rangle\left\langle s m_{2}^{\prime}+m_{3}^{\prime} \frac{1}{2} m_{1}^{\prime} \mid S m_{1}^{\prime}+m_{2}^{\prime}+m_{3}^{\prime}\right\rangle \\
& \left\langle\frac{1}{2} \nu_{2}^{\prime} \frac{1}{2} \nu_{3}^{\prime} \mid t \nu_{2}^{\prime}+\nu_{3}^{\prime}\right\rangle\left\langle t \nu_{2}^{\prime}+\nu_{3}^{\prime} \frac{1}{2} \nu_{1}^{\prime} \mid T \nu_{1}^{\prime}+\nu_{2}^{\prime}+\nu_{3}^{\prime}\right\rangle\left\langle L M_{L} S m_{1}^{\prime}+m_{2}^{\prime}+m_{3}^{\prime} \mid J M_{J}\right\rangle \\
& Y_{l \lambda}^{L M_{L}}\left(\hat{p}_{4}, \hat{q}_{4}\right) G_{0}\left(p_{4}, q_{4}\right)\left\langle p_{4}, q_{4}, \alpha|T| \Phi\right\rangle .
\end{aligned}
$$

For channels $\alpha \neq \alpha_{d}$ the only singularity is the $G_{0}$ singularity. When $\vec{p}_{4}$ and $\vec{q}_{4}$ are taken as an integration variables in (D4) $G_{0}$ becomes

$$
G_{0}\left(p_{4}, q_{4}\right)=G_{0}\left(p^{\prime}, q\right)=\frac{1}{E+i \epsilon-\frac{1}{m}\left(p^{\prime 2}+\frac{3}{4} q^{2}\right)}
$$

and the contribution to the matrix element $\left\langle\vec{p} \vec{q} m_{1} m_{2} m_{3} \nu_{1} \nu_{2} \nu_{3}\left|t_{c}^{R} P G_{0} T\right| \Phi\right\rangle$ coming from these channels is given by

$$
\left.\left\langle\vec{p} \vec{q} m_{1} m_{2} m_{3} \nu_{1} \nu_{2} \nu_{3}\left|t_{c}^{R} P G_{0} T\right| \Phi\right\rangle\right|_{\alpha \neq \alpha_{d}}=\delta_{\nu_{2},+\frac{1}{2}} \delta_{\nu_{3},+\frac{1}{2}} \int p^{\prime 2} d p^{\prime} \frac{1}{E+i \varepsilon-\frac{p^{\prime 2}}{m}-\frac{3 q^{2}}{4 m}} \int d \phi_{p^{\prime}} d\left(\cos \theta_{p^{\prime}}\right)
$$




$$
\begin{aligned}
& t_{c}^{R}\left(\vec{p}, \vec{p}^{\prime} ; E(q)\right) \sum_{\alpha \neq \alpha_{d} L S} \sqrt{\hat{j} \hat{I} \hat{L} \hat{S}}\left\{\begin{array}{ccc}
l & s & j \\
\lambda & \frac{1}{2} & I \\
L & S & J
\end{array}\right\}\left\langle L M_{L} S m_{1}+m_{2}+m_{3} \mid J m_{p}+m_{d}\right\rangle \\
& {\left[\left\langle\frac{1}{2} m_{3} \frac{1}{2} m_{1} \mid s m_{3}+m_{1}\right\rangle\left\langle s m_{3}+m_{1} \frac{1}{2} m_{2} \mid S m_{1}+m_{2}+m_{3}\right\rangle\right.} \\
& \left\langle\frac{1}{2} \nu_{3} \frac{1}{2} \nu_{1} \mid t \nu_{3}+\nu_{1}\right\rangle\left\langle t \nu_{3}+\nu_{1} \frac{1}{2} \nu_{2} \mid T \nu_{1}+\nu_{2}+\nu_{3}\right\rangle \\
& \times Y_{l \lambda}^{L M_{L}}\left(-\frac{1}{2} \vec{p}^{\prime}-\frac{3}{4} \vec{q}, \vec{p}^{\prime}-\frac{1}{2} \vec{q}\right)\left\langle\left|-\frac{1}{2} \vec{p}^{\prime}-\frac{3}{4} \vec{q}\right|,\left|\vec{p}^{\prime}-\frac{1}{2} \vec{q}\right|, \alpha|T| \Phi\right\rangle \\
& +\left\langle\frac{1}{2} m_{1} \frac{1}{2} m_{2} \mid s m_{1}+m_{2}\right\rangle\left\langle s m_{1}+m_{2} \frac{1}{2} m_{3} \mid S m_{1}+m_{2}+m_{3}\right\rangle \\
& \left\langle\frac{1}{2} \nu_{1} \frac{1}{2} \nu_{2} \mid t \nu_{1}+\nu_{2}\right\rangle\left\langle t \nu_{1}+\nu_{2} \frac{1}{2} \nu_{3} \mid T \nu_{1}+\nu_{2}+\nu_{3}\right\rangle \\
& \left.\times Y_{l \lambda}^{L M_{L}}\left(-\frac{1}{2} \vec{p}^{\prime}+\frac{3}{4} \vec{q},-\vec{p}^{\prime}-\frac{1}{2} \vec{q}\right)\left\langle\left|-\frac{1}{2} \vec{p}^{\prime}+\frac{3}{4} \vec{q}\right|,\left|-\vec{p}^{\prime}-\frac{1}{2} \vec{q}\right|, \alpha|T| \Phi\right\rangle\right],
\end{aligned}
$$

where $m_{p}$ and $m_{d}$ are spin projections of the incoming proton and deuteron, and $M_{L}=m_{p}+m_{d}-$ $m_{1}-m_{2}-m_{3}$. Integration over $p^{\prime}$ can be performed numerically taking care of the pole in $G_{0}$ by e.g. the subtraction method.

For $\alpha_{d}$ channels one can decompose $G_{0}\left(p_{4}, q_{4}\right)\left\langle p_{4} q_{4} \alpha_{d}|T| \Phi\right\rangle$ in (D4) in the following way

$$
\begin{aligned}
& G_{0}\left(p_{4}, q_{4}\right)\left\langle p_{4} q_{4} \alpha_{d}|T| \Phi\right\rangle=\frac{1}{\frac{3}{4 m}\left(q_{\text {max }}^{2}-q_{4}^{2}\right)-\frac{1}{m} p_{4}^{2}+i \epsilon} \frac{\left\langle p_{4} q_{4} \alpha_{d}|\hat{T}| \Phi\right\rangle}{\frac{3}{4 m}\left(q_{0}^{2}-q_{4}^{2}\right)+i \epsilon} \\
& \frac{1}{\frac{3}{4 m}\left(q_{\max }^{2}-q_{4}^{2}\right)-\frac{1}{m} p_{4}^{2}+i \epsilon} \frac{\left\langle p_{4} q_{4} \alpha_{d}|\hat{T}| \Phi\right\rangle}{\left|E_{d}\right|+\frac{1}{m} p_{4}^{2}}-\frac{1}{\frac{3}{4 m}\left(q_{0}^{2}-q_{4}^{2}\right)+i \epsilon} \frac{\left\langle p_{4} q_{4} \alpha_{d}|\hat{T}| \Phi\right\rangle}{\left|E_{d}\right|+\frac{1}{m} p_{4}^{2}} .
\end{aligned}
$$

The first part in (D7) can be integrated in the same way as the contribution from channels $\alpha \neq \alpha_{d}$, resulting in expression (마) with $\langle p q \alpha|T| \Phi\rangle$ replaced by $\frac{\left\langle p q \alpha_{d}|\hat{T}| \Phi\right\rangle}{\left|E_{d}\right|+\frac{1}{m} p^{2}}$.

Calculating contribution from the second term in (D7) one takes in (D3) $\vec{p}^{\prime}$ and $\vec{p}_{4}$ as an integration variables. This gives the following result for that term

$$
\begin{aligned}
& \left\langle\vec{p} \vec{q} m_{1} m_{2} m_{3} \nu_{1} \nu_{2} \nu_{3}\left|t_{c}^{R} P G_{0} T\right| \Phi\right\rangle||_{\alpha_{d}}^{\text {second }}=-\delta_{\nu_{2},+\frac{1}{2}} \delta_{\nu_{3},+\frac{1}{2}} \int q_{4}^{2} d q_{4} \frac{1}{\frac{3}{4 m}\left(q_{0}^{2}-q_{4}^{2}\right)+i \varepsilon} \int d \phi_{q_{4}} d\left(\cos \theta_{q_{4}}\right) \\
& \sum_{\alpha_{d} L S} \sqrt{\hat{\hat{j} \hat{I} \hat{L} \hat{S}}}\left\{\begin{array}{ccc}
l & s & j \\
\lambda & \frac{1}{2} & I \\
L & S & J
\end{array}\right\}\left\langle L M_{L} S m_{1}+m_{2}+m_{3} \mid J m_{p}+m_{d}\right\rangle \\
& {\left[\left\langle\frac{1}{2} m_{3} \frac{1}{2} m_{1} \mid s m_{3}+m_{1}\right\rangle\left\langle s m_{3}+m_{1} \frac{1}{2} m_{2} \mid S m_{1}+m_{2}+m_{3}\right\rangle\right.} \\
& \left\langle\frac{1}{2} \nu_{3} \frac{1}{2} \nu_{1} \mid t \nu_{3}+\nu_{1}\right\rangle\left\langle t \nu_{3}+\nu_{1} \frac{1}{2} \nu_{2} \mid T \nu_{1}+\nu_{2}+\nu_{3}\right\rangle t_{c}^{R}\left(\vec{p}, \frac{1}{2} \vec{q}+\overrightarrow{q_{4}} ; E(q)\right) \\
& \times Y_{l \lambda}^{L M_{L}}\left(-\vec{q}-\frac{1}{2} \vec{q}_{4}, \hat{q_{4}}\right) \frac{\left\langle\left|-\vec{q}-\frac{1}{2} \overrightarrow{q_{4}}\right|, q_{4}, \alpha_{d}|\hat{T}| \Phi\right\rangle}{\left|E_{d}\right|+\frac{1}{m}\left|\vec{q}+\frac{1}{2} \vec{q}_{4}\right|^{2}}
\end{aligned}
$$




$$
\begin{aligned}
& +\left\langle\frac{1}{2} m_{1} \frac{1}{2} m_{2} \mid s m_{1}+m_{2}\right\rangle\left\langle s m_{1}+m_{2} \frac{1}{2} m_{3} \mid S m_{1}+m_{2}+m_{3}\right\rangle \\
& \left\langle\frac{1}{2} \nu_{1} \frac{1}{2} \nu_{2} \mid t \nu_{1}+\nu_{2}\right\rangle\left\langle t \nu_{1}+\nu_{2} \frac{1}{2} \nu_{3} \mid T \nu_{1}+\nu_{2}+\nu_{3}\right\rangle t_{c}^{R}\left(\vec{p},-\frac{1}{2} \vec{q}-\vec{q}_{4} ; E(q)\right) \\
& \left.\times Y_{l \lambda}^{L M_{L}}\left(\vec{q}+\frac{1}{2} \vec{q}_{4}, \hat{q}_{4}\right) \frac{\left\langle\left|\vec{q}+\frac{1}{2} \vec{q}_{4}\right|, q_{4}, \alpha_{d}|\hat{T}| \Phi\right\rangle}{\left|E_{d}\right|+\frac{1}{m}\left|\vec{q}+\frac{1}{2} \vec{q}_{4}\right|^{2}}\right]
\end{aligned}
$$

Again one has a simple pole which can be treated using e.g. the subtraction method.

For the exclusive breakup the calculation of the contributions (D6) and (D8) must be performed for each complete geometry in a coordinate system used when calculating $<p q \alpha|T| \Phi>$ where the z- axis was taken to be parallel to the incoming proton momentum.

For the elastic scattering an additional integration has to be done providing the following contribution to the elastic scattering amplitude from the $<\Phi^{\prime}\left|P t_{c}^{R} P G_{0}\right| \alpha^{\prime}><\alpha^{\prime}|T| \Phi>$ term:

$$
\begin{aligned}
& \left.<\Phi^{\prime}\left|P t_{c}^{R} P G_{0}\right| \alpha^{\prime}><\alpha^{\prime}|T| \Phi\right\rangle=\left[-\int d \vec{q} \sum_{m_{1} m_{2}} \sum_{L M} \varphi_{L}\left(\left|\vec{q}+\frac{1}{2} \vec{q}_{0}{ }^{\prime}\right|\right) Y_{L, M}^{*}\left(\vec{q}+\frac{\hat{1}}{2} \vec{q}_{0}{ }^{\prime}\right)\right. \\
& \left\langle L 11, M, m_{d}^{\prime}-M, m_{d}^{\prime}\right\rangle\left\langle\frac{1}{2} \frac{1}{2} 1, m_{1} m_{2} m_{d}^{\prime}-M\right\rangle \frac{\sqrt{2}}{2} \\
& \left\langle-\frac{1}{2} \vec{q}-\vec{q}_{0}^{\prime}, \vec{q} m_{1} m_{2} m_{N^{\prime}}, \nu_{1}=-\frac{1}{2} \nu_{2}=+\frac{1}{2} m_{t_{N^{\prime}}}=+\frac{1}{2}\left|t_{c}^{R} P G_{0} T\right| \Phi\right\rangle \\
& +\sum_{m_{1} m_{3}} \sum_{L M} \varphi_{L}\left(\left|-\vec{q}-\frac{1}{2} \vec{q}_{0}{ }^{\prime}\right|\right) Y_{L, M}^{*}\left(-\vec{q}-\frac{1}{2} \vec{q}_{0}{ }^{\prime}\right) \\
& \left\langle L 11, M, m_{d}^{\prime}-M, m_{d}^{\prime}\right\rangle\left\langle\frac{1}{2} \frac{1}{2} 1, m_{3} m_{1} m_{d}^{\prime}-M\right\rangle \frac{\sqrt{2}}{2} \\
& \left\langle\frac{1}{2} \vec{q}+\vec{q}_{0}{ }^{\prime}, \vec{q} m_{1} m_{N^{\prime}} m_{3}, \nu_{1}=-\frac{1}{2} m_{t_{N^{\prime}}}=+\frac{1}{2} \nu_{3}=+\frac{1}{2}\left|t_{c}^{R} P G_{0} T\right| \Phi\right\rangle .
\end{aligned}
$$

[1] E. O. Alt, W. Sandhas, and H. Ziegelmann, Phys. Rev. C 17, 1981 (1978).

[2] E. O. Alt and W. Sandhas, in Coulomb Interactions in Nuclear and Atomic Few-Body Collisions, ed. by F.S. Levin and D. Micha (Plenum, New York 1996), p.1.

[3] E. O. Alt and M. Rauh, Phys. Rev. C49, R2285 (1994).

[4] E. O. Alt, A. M. Mukhamedzhanov, M. M. Nishonov, and A. I. Sattarov, Phys. Rev. C65, 064613 (2002).

[5] W. Glöckle, H. Witała, D. Hüber, H. Kamada, J. Golak, Phys. Rep. 274, 107 (1996).

[6] E. Epelbaum, Prog. Part. Nucl. Phys. 57, 654 (2006); and references therein.

[7] A. Kievsky, M. Viviani, and S. Rosati, Phys. Rev. C 52, R15 (1995).

[8] A. Deltuva, A. C. Fonseca, and P. U. Sauer, Phys. Rev. C72, 054004 (2005).

[9] A. Deltuva, A. C. Fonseca, and P. U. Sauer, Phys. Rev. C71, 054005 (2005).

[10] E. Stephan et al., Phys. Rev. C76, 057001 (2007). 
[11] G. Rauprich et al., Nucl. Phys. A535, 313 (1991).

[12] R. Großmann et al., Nucl. Phys. A603, 161 (1996).

[13] H. Patberg et al., Phys. Rev. C53, 1497 (1996).

[14] M. Allet et al., Few-Body Syst. C20, 27 (1996).

[15] J. Zejma et al., Phys. Rev. C55, 42 (1997).

[16] R. Skibiński, J. Golak, H. Witała, and W.Glöckle, Eur. Phys. Journal A40, 215 (2009).

[17] H. Witała, W. Glöckle, H.Kamada, Phys. Rev. C43, 1619 (1991).

[18] W. Glöckle, The Quantum Mechanical Few-Body Problem, Springer Verlag 1983.

[19] J.C.Y. Chen and A.C. Chen, in Advances of Atomic and Molecular Physics, edited by D. R. Bates and J. Estermann ( Academic, New York, 1972), Vol. 8.

[20] R. Machleidt, F. Sammarruca, and Y. Song, Phys. Rev. C53, R1483 (1996).

[21] W. Grüebler et al., Nucl. Phys. A398, 445 (1983). 


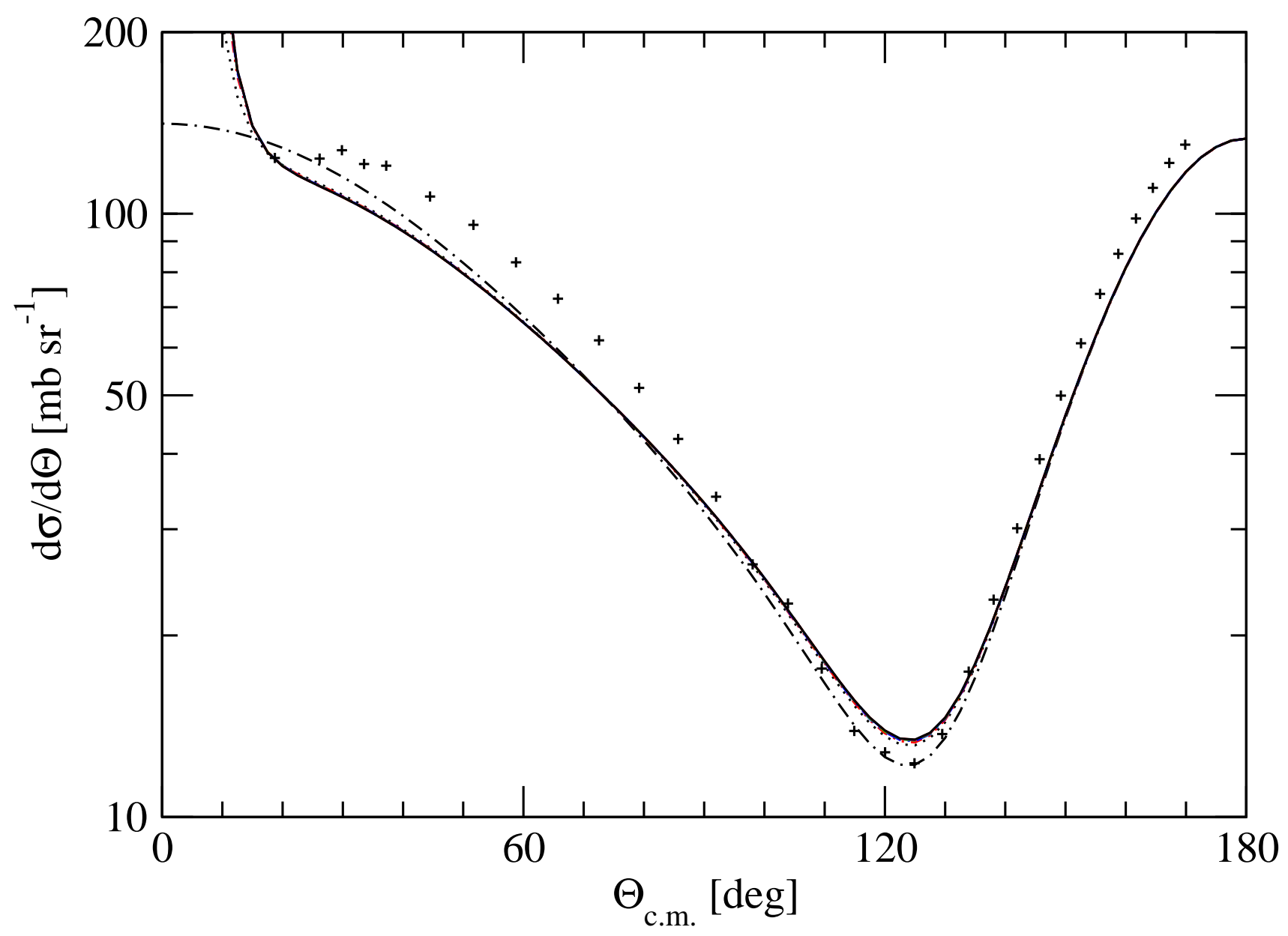

FIG. 1: (color online) The convergence in the cut-off radius $\mathrm{R}$ of the pd elastic scattering cross section $\frac{d \sigma}{d \Omega}$ shown as a function of the c.m. angle $\Theta_{c . m}$. at the incoming proton energy $E_{p}^{l a b}=13 \mathrm{MeV}$. These cross sections were calculated with the screened Coulomb force and the CD Bonn nucleon-nucleon potential [20] restricted to the ${ }^{1} S_{0}$ and ${ }^{3} S_{{ }^{-}}{ }^{3} D_{1}$ partial waves. The screening radii are $: R=20 \mathrm{fm}$ (black dotted line), $R=40 \mathrm{fm}$ (green double-dashed -dotted line), $R=60 \mathrm{fm}$ (blue long-dashed-dotted line), $R=80 \mathrm{fm}$ (red dashed- double-dotted line), $R=100 \mathrm{fm}$ (blue short-dashed line), $R=120 \mathrm{fm}$ (red long-dashed line), $R=140 \mathrm{fm}$ (black solid line). The $R=40-140 \mathrm{fm}$ lines are practically overlapping. The black dasheddotted line is the corresponding nd elastic scattering cross section. The pluses are $E_{p}^{l a b}=12 \mathrm{MeV}$ pd elastic scattering cross section data of Ref. [21]. 


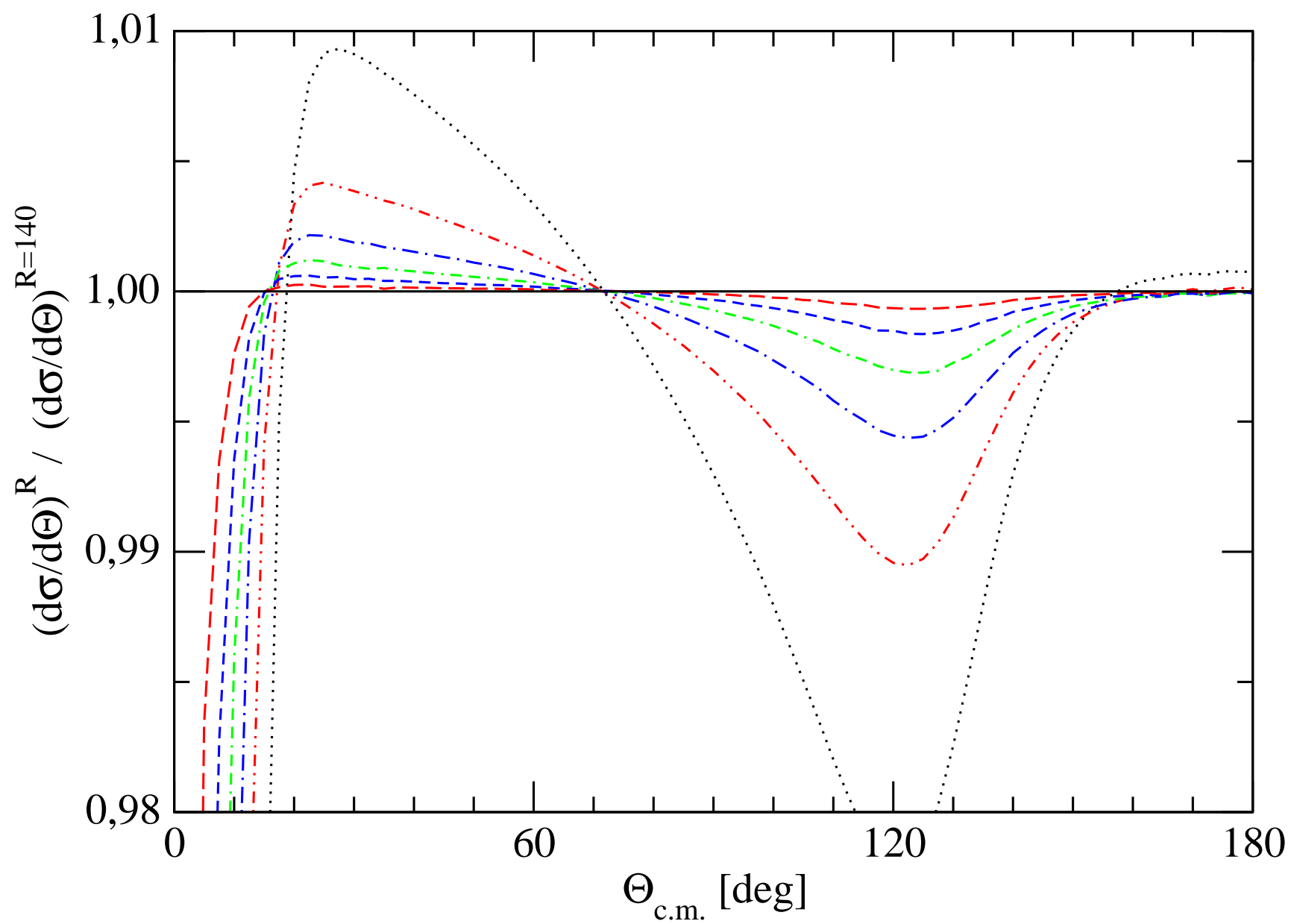

FIG. 2: (color online) The convergence in the cut-off radius $\mathrm{R}$ of the pd elastic scattering cross section $\frac{d \sigma}{d \Omega}$ at the incoming proton energy $E_{p}^{l a b}=13 \mathrm{MeV}$, shown as the ratio $\frac{d \sigma}{d \Omega}^{R} / \frac{d \sigma}{d \Omega}^{R=140}$. For the description of the lines see Fig. 1 . 


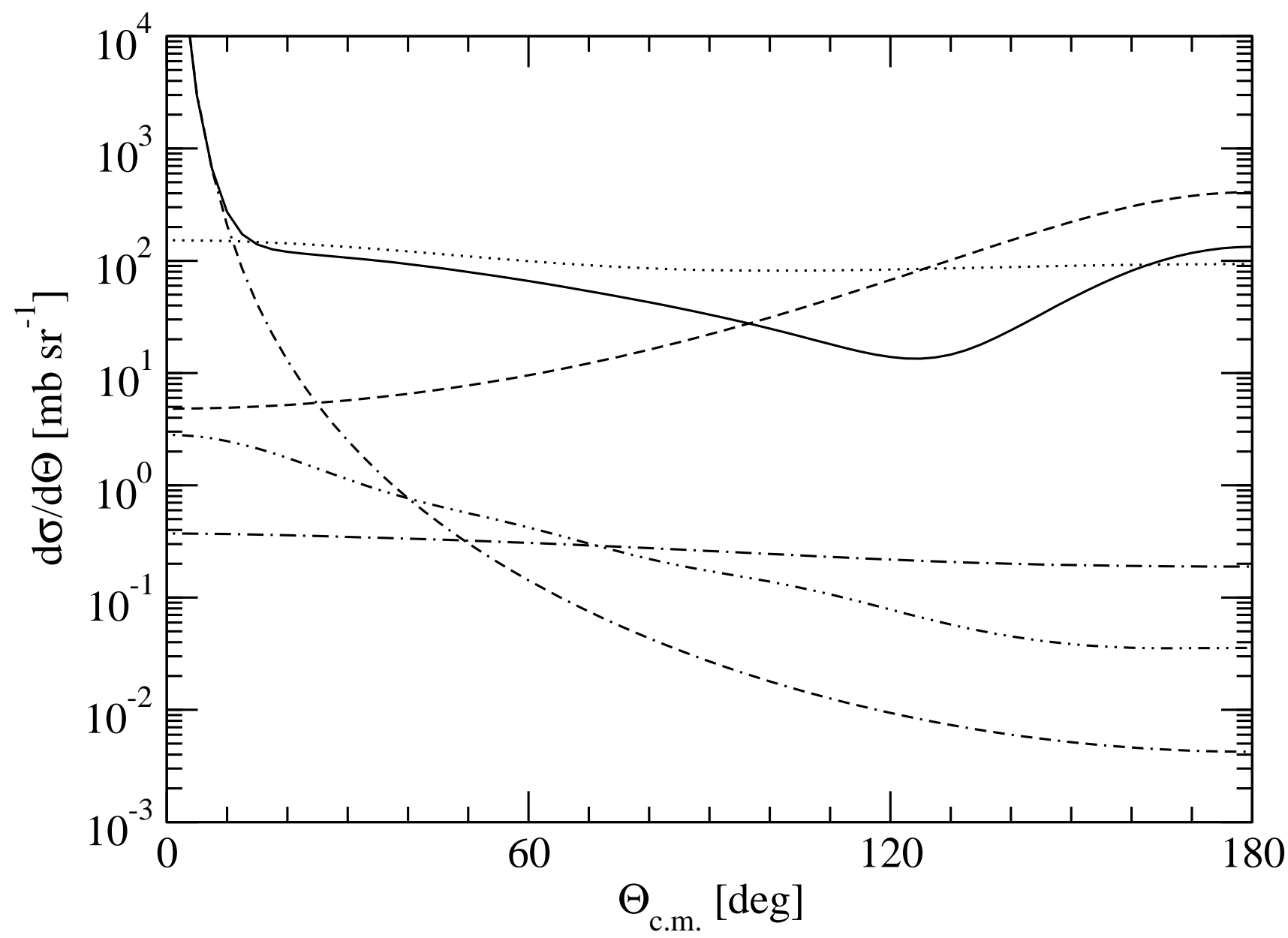

FIG. 3: The contributions of different terms to the pd elastic scattering cross section $\frac{d \sigma}{d \Omega}$ at the incoming proton energy $E_{p}^{l a b}=13 \mathrm{MeV}$ calculated with the screening radius $R=100 \mathrm{fm}$. The dotted and dashed lines are contributions of the $\left\langle\Phi^{\prime}|P T| \Phi\right\rangle$ and $\left\langle\Phi^{\prime}\left|P G_{0}^{-1}\right| \Phi\right\rangle$ terms, respectively. The double-dashed-dotted line is the contribution of the $\left\langle\Phi^{\prime}\left|P t_{c}^{R} P\right| \Phi\right\rangle$ term coming with the 3-dimensional screened Coulomb t-matrix $t_{c}^{R}$. The dashed-double-dotted and dashed-dotted lines are contributions of the $\left\langle\Phi^{\prime}\left|P t_{c}^{R} P\right| \Phi\right\rangle$ and $\left\langle\Phi^{\prime}\left|P t_{c}^{R} P G_{0} T\right| \Phi\right\rangle$ terms, respectively, which are calculated with the partial-wave decomposed screened Coulomb t-matrix. The solid line encompasses all terms. In this feasibility study the 3 -dimensional $t_{c}^{R}$ is replaced by $V_{c}^{R}$. 

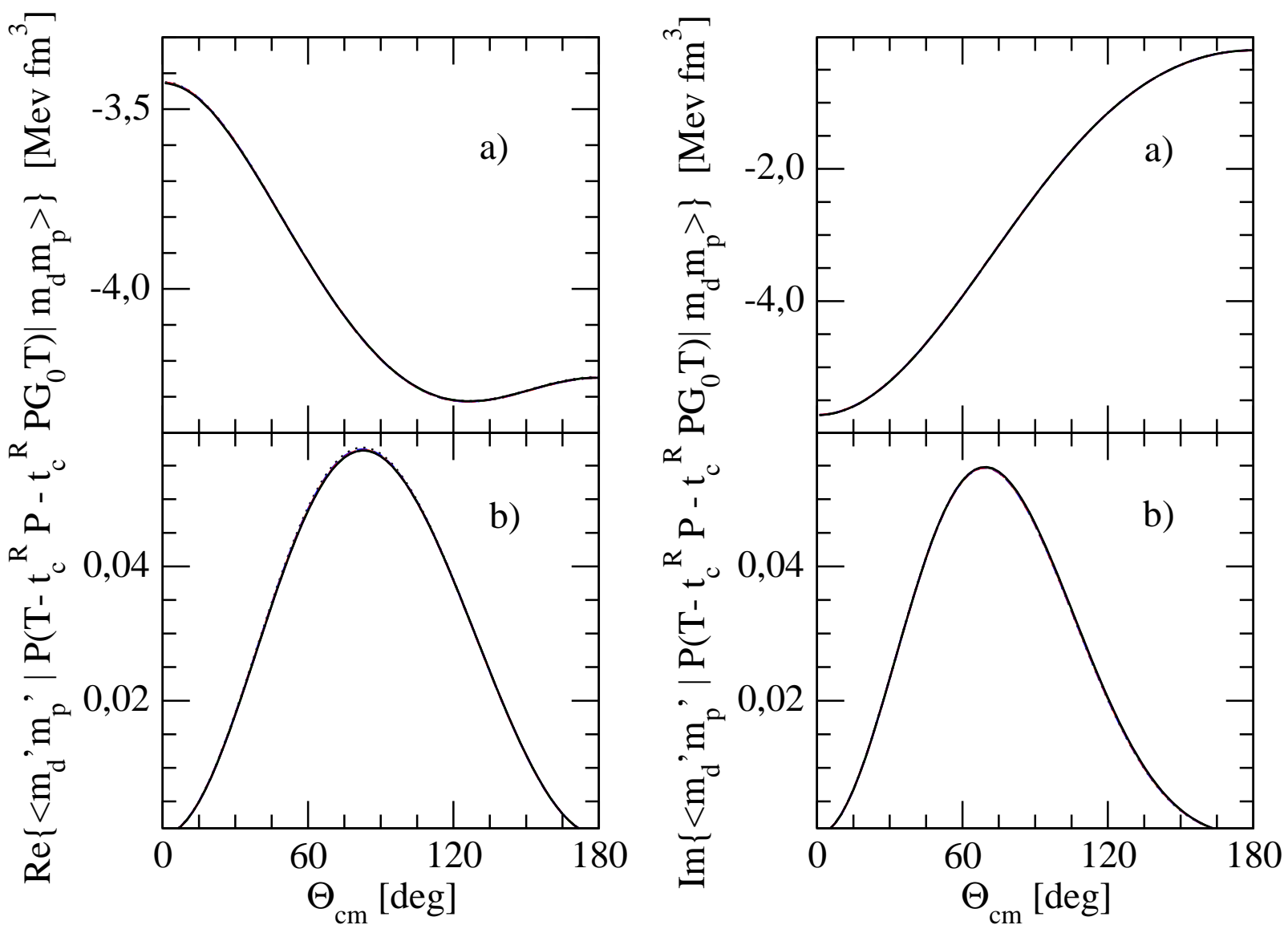

FIG. 4: (color online) The independence of the real (left column) and imaginary (right column) parts of the partial wave contribution $\left\langle\Phi_{m_{d^{\prime}} m_{p^{\prime}}}^{\prime}\left|P\left(T-t_{c}^{R} P-t_{c}^{R} P G_{0} T\right)\right| \Phi_{m_{d} m_{p}}\right\rangle$ to the $E_{p}^{l a b}=13 \mathrm{MeV}$ pd elastic scattering transition amplitude on the cut-off radius $\mathrm{R}$. The different lines are: $R=20 \mathrm{fm}$ - dotted, $R=40 \mathrm{fm}$ - short-dashed, $R=60 \mathrm{fm}$ - long-dashed, $R=80 \mathrm{fm}$ - short-dashed-dotted, $R=100 \mathrm{fm}$ - long-dashed-dotted, $R=120 \mathrm{fm}$ - double-dotted-dashed, $R=140 \mathrm{fm}$ - solid. All the lines are practically overlapping. The incoming and outgoing deuteron and proton spin projections are for a): $m_{d}=m_{d^{\prime}}=-1$ and $m_{p}=m_{p^{\prime}}=-\frac{1}{2}$ and for b): $m_{d}=-1, m_{d^{\prime}}=+1$, and $m_{p}=m_{p^{\prime}}=+\frac{1}{2}$. 